\title{
Effective action and collective modes in quasi-one-dimensional spin-density-wave systems
}

\author{
K. Sengupta ${ }^{(1)}$ and N. Dupuis ${ }^{(2)}$ \\ (1) Department of Physics, University of Maryland, College Park, MD 20742-4111, USA \\ (2) Laboratoire de Physique des Solides, Université Paris-Sud, 91405 Orsay, France
}

(January 24, 2000)

\begin{abstract}
We derive the effective action describing the long-wavelength low-energy collective modes of quasione-dimensional spin-density-wave (SDW) systems, starting from the Hubbard model within weak coupling approximation. The effective action for the spin-wave mode corresponds to an anisotropic non-linear sigma model together with a Berry phase term. We compute the spin stiffness and the spin-wave velocity. We also obtain the effective action for the sliding mode (phason) taking into account the density fluctuations from the outset and in presence of a weak external electromagnetic field. This leads to coupled equations for the phase of the SDW condensate and the charge density fluctuations. We also calculate the conductivity and the density-density correlation function.
\end{abstract}

PACS Numbers:72.15.Nj, 75.30.Fv

\section{INTRODUCTION}

Materials with highly anisotropic crystal structure often exhibit density-wave (DW) instabilities at low temperature 1 While inorganic linear chain compounds usually develop a charge-density-wave (CDW) instability, several group of organic conductors present a SDW ground state. Well known examples include transition metal bronzes such as $\mathrm{K}_{0.3} \mathrm{MoO}_{3}$ for CDW systems, and the Bechgaard salts (TMTSF $)_{2} \mathrm{X}\left(\mathrm{X}=\mathrm{ClO}_{4}, \mathrm{PF}_{6}\right)$ for SDW systems.

In quasi-one-dimensional (quasi-1D) systems, DW instabilities result from nesting properties of the Fermi surface. Indeed, in this case the particle-hole response function exhibits a logarithmic singularity $\sim \ln \left(E_{0} / T\right)$, where $E_{0}$ is an ultra-violet cutoff of the order of the bandwidth. In presence of repulsive electron-electron or electron-phonon interactions, this leads to an instability of the metallic phase at low temperature. CDW ground states resulting from electron-phonon interaction were first discussed by Fröhlich and Peierls, sibility of a SDW ground state due to repulsive electron electron interaction was first postulated by Overhauser.

In the SDW ground state, quasi-particle excitations exhibit a gap $2 \Delta_{0}$, where $\Delta_{0}$ is the SDW order parameter. The low-energy behavior of the system is then dominated by low-lying collective modes. In presence of an incommensurate SDW, two continuous symmetries are spontaneously broken: the translational symmetry and the rotational symmetry in spin space. This leads to the existence of two gapless Goldstone modes: a sliding mode (phason) and a spin-wave mode (magnon). Contrary to the case of superconductors, collective modes in DW systems directly couple to external fields, so that they can easily be observed in various experiments. For instance, the non-linear dc conductivity is a manifestation of the existence of a phason mode which is pinned by impurities in real systems.
In CDW systems, collective modes were first studied by Lee, Rice and Anderson $\mathrm{O}$ From the computation of the Green functions, they deduced the existence of a gapped amplitude mode and a gapless phase mode (phason). The latter corresponds to a sliding of the CDW and would lead to an infinite conductivity in a clean system (i.e. with no impurity) as first proposed by Fröhlich.2 Fukuyama then proposed an effective phase Iagrangian $\mathcal{L}(\theta)$ determining the dynamics of the phason, 9 where the condensate phase $\theta$ determines the position of the DW with respect to the underlying crystal lattice. The first attempt to derive $\mathcal{f}(\theta)$ rigorously is due to Brazovskii and Dzyaloshinskii. The phase Lagrangian was latter used to study the interaction of the CDW with impurities and the mechanism of pinning/depinning which is at the origin of the non-linear dc conductivity observed in transport experiments.

To a large extent, the analysis of collective modes in CDW systems can be transposed to SDW systems 100 The amplitude mode has a gap $2 \Delta_{0}$ and is therefore strongly damped due to the coupling with quasi-particle excitations above the mean-field gap 10 As in CDW systems, the phason corresponds to a sliding of the DW and leads to an infinite Fröhlich conductivity in the absence of impurities. However, since the SDW instability is driven by electron-electron interactions, the coupling to the lattice plays no role and its mass is not renormalized by phonons. The pinning by impurities is also weaker, since the SDW couples to charge inhomogeneities only to second order. The spin-wave mode, which is specific to SDW system is also obtained from the poles of the Green functions. 14

The functional integral formalism has also proven useful to study collective modes in DW systems. 1724 The main advantage of this formalism is that it allows to derive the effective Lagrangian of the Goldstone modes from first principles. Both the phase Lagrangian $\mathcal{L}(\theta)$ and the effective Lagrangian for the spin-wave mode can be ob- 
tained within this formalism.

In this paper we derive the effective action describing the low-energy collective modes of quasi-1D SDW systems, starting from the Hubbard model with weak on-site interaction. This effective action describes the behavior of the system at energies much smaller than the meanfield gap $\Delta_{0}$ or, equivalently, at length scales much larger than the mean-field coherence lengths $v_{F} / \Delta_{0}$ and $v_{\perp} / \Delta_{0}$ $\left(v_{F}\right.$ and $v_{\perp}$ are the velocities along and across the conducting chains). The improvement with respect to previous works is twofold. First, we show that the effective action for the spin-wave mode is given by an anisotropic non-linear sigma model (NL $\sigma \mathrm{M})$ together with a Berry phase term. Such a result is known for the isotropic 2D Hubbard model, 55 but has not been derived for weakly coupled chain systems. Instead, it is generally assumed that the spin dynamics can be deduced from an effective Heisenberg Hamiltonian. Second, we introduce the longwavelength charge-density field from the outset. We thus obtain an effective Lagrangian $\mathcal{L}(\theta, \rho)$ which is a functional of two independent fields: $\theta$, which is the phase of the SDW condensate and the charge-density field $\rho$. This yields coupled equations of motion for $\theta$ and $\rho$. The interaction between these two quantities leads to a renormalization of the longitudinal phason velocity.

The first step in the functional integral formalism is to introduce auxiliary fields describing spin and charge fluctuations. The main technical difficulty is then to recover the mean-field (or Hartree-Fock) solution in a saddlepoint approximation, while maintaining rotational invariance in spin space which is a necessary condition for obtaining the spin-wave mode. To overcome this difficulty, we introduce a space and time fluctuating spin-quantization axis, following a method introduced by Schulz26 and Weng et al 27 for the isotropic 2D Hubbard model. Note that in quasi-1D systems, the distinction between right and left moving electrons allows one to write the Hamiltonian in a rotationally invariant form which is well suited for the calculation of the spin-wave mode if one focuses only on the $2 k_{F}$ particle-hole (Peierls) channel.28 However, the concomitant consideration of the Landau channel (long-wavelength charge fluctuations), which is at the heart of our approach, does require the introduction of a fluctuating spin-quantization axis.

The organization of the paper is as follows. The effective action of the system is derived in Sec. III. We first introduce bosonic fields describing charge and spin fluctuations, and the fluctuating spin-quantization axis. We take special care to introduce the physical charge density field $\rho$. The standard mean-field theory is recovered in Sec. II A, while fluctuations are studied in Sec. II B. The latter are most conveniently computed by performing a chiral rotation of the Fermion fields. The corresponding Jacobian (the so-called chiral anomaly) is calculated in Sec. II B 2.

The effective action governing the dynamics of the spin-wave mode is shown to be a NL $\sigma \mathrm{M}$ together with a topological Berry phase term in Sec. III. We explicitly calculate the spin stiffness, the spin-wave velocity, and the coupling constant of the NL $\sigma \mathrm{M}$.

The sliding mode is studied in Sec. IV. We obtain coupled equations of motion for the phase $\theta$ of the SDW condensate and the charge fluctuations $\rho$. By integrating out one of these fields, we obtain the effective action as a functional of either $\theta$ or $\rho$. We also calculate the conductivity and the density-density correlation function from the effective action.

We do not consider long-range Coulomb interaction, which would require taking into account normal electrons that are thermally excited above the gap. The latter are indeed expected to play a crucial role in the screening of the interaction.29 Note however that the Coulomb interaction affects neither the spin-wave mode nor the transverse phason mode sampled by optical spectroscopy.

We consider only the zero-temperature limit, and take $\hbar=k_{B}=1$ throughout the paper.

\section{EFFECTIVE ACTION}

In the vicinity of the Fermi level, the electron dispersion is well approximated as

$$
\epsilon\left(k_{x}, k_{y}\right)=v_{F}\left(\left|k_{x}\right|-k_{F}\right)-2 t_{b} \cos \left(k_{y} b\right)
$$

where $k_{x}$ and $k_{y}$ are the electron momenta along and across the conducting chains, $t_{b}$ is the transfer integral in the transverse direction, and $b$ the inter-chain distance. In Eq. (2.1), the longitudinal dispersion is linearized around the $1 \mathrm{D}$ Fermi points $\pm k_{F}$ and $v_{F}=$ $2 a t_{a} \sin \left(k_{F} a\right)$ is the corresponding Fermi velocity, with $t_{a} \gg t_{b}$ being the transfer integral and $a$ the lattice spacing along the chains. The linearized dispersion (2.1) satisfies the property $\epsilon(\mathbf{k})=-\epsilon(\mathbf{k}+\mathbf{Q})$, which corresponds to a perfect nesting of the Fermi surface at wave vector $\mathbf{Q}=\left(2 k_{F}, \pi / b\right)$. Actually this property is an artifact of the linearization and does not hold for the original tight-binding dispersion unless the system is half-filled $\left(k_{F}=\pi / 2 a\right)$. Deviations from perfect nesting can be taken into account by adding higher harmonics to the transverse dispersion $-2 t_{b} \cos \left(k_{y} b\right)$. For simplicity, we shall not consider such terms and restrict ourselves to the perfect nesting case. We also assume that the SDW is incommensurate with the crystal lattice, so that there is no pinning by the lattice.

Following the standard procedure, we introduce right and left Fermionic fields $\psi_{+\sigma}$ and $\psi_{-\sigma}$ ( $\sigma$ is the spin index). In terms of the quartet of Fermion fields $\psi^{\dagger}=$ $\left(\psi_{+\uparrow}^{\dagger}, \psi_{-\uparrow}^{\dagger}, \psi_{+\downarrow}^{\dagger}, \psi_{-\downarrow}^{\dagger}\right)$, one can write the Hamiltonian of the system as $H=H_{0}+H_{I}$, where

$$
\begin{aligned}
H_{0}= & \sum_{n} \int d x\left[\psi_{n}^{\dagger}(x) v_{F}\left(-i \partial_{x} \tau_{3}-k_{F}\right) \psi_{n}(x)\right. \\
& \left.-t_{b} \sum_{\delta= \pm 1} \psi_{n}^{\dagger}(x) \psi_{n+\delta}(x)\right]
\end{aligned}
$$




$$
H_{I}=U \sum_{n} \int d x \psi_{n \uparrow}^{\dagger}(x) \psi_{n \downarrow}^{\dagger}(x) \psi_{n \downarrow}(x) \psi_{n \uparrow}(x),
$$

$U$ is the on-site Coulomb interaction strength and $n$ the chain index. Here and in the following, $\tau_{\mu}(\mu=1,2,3)$ and $\sigma_{\nu}(\nu=x, y, z)$ are $2 \times 2$ Pauli matrices acting on left/right and spin indices of the Fermionic fields respectively. The product $\tau_{\mu} \sigma_{\nu}$ is to be understood as direct product of the matrices $\tau_{\mu}$ and $\sigma_{\nu}$, and any single matrix $\tau_{\mu}$ or $\sigma_{\nu}$ as direct product of that matrix with the unit matrix.

Introducing the charge and spin density fields

$$
\begin{aligned}
\rho_{c} & =\psi^{\dagger} \psi, \quad \rho_{s}=\psi^{\dagger} \sigma_{z} \psi, \\
\rho_{c+} & =\psi^{\dagger} \tau_{-} \psi, \quad \rho_{s+}=\psi^{\dagger} \tau_{-} \sigma_{z} \psi,
\end{aligned}
$$

where $\tau_{ \pm}=\left(\tau_{1} \pm i \tau_{2}\right) / 2$, we rewrite the interaction Hamiltonian as

$$
\begin{aligned}
H_{I}= & \frac{U}{4} \sum_{n} \int d x\left[\left(\rho_{c n}(\tau, x)\right)^{2}-\left(\rho_{s n}(\tau, x)\right)^{2}\right. \\
& +2\left(\rho_{c+n}^{*}(\tau, x) \rho_{c+n}(\tau, x)\right. \\
& \left.\left.-\rho_{s+n}^{*}(\tau, x) \rho_{s+n}(\tau, x)\right)\right] .
\end{aligned}
$$

In the Matsubara formalism, the partition function of the system can be written as a functional integral over anticommuting Grassmann variables, $Z=$ $\int D \psi^{\dagger} D \psi e^{-S\left[\psi^{\dagger}, \psi\right]}$, with the action

$$
S=\int_{0}^{\beta} d \tau\left[\sum_{n} \int d x \psi_{n}^{\dagger}(\tau, x) \partial_{\tau} \psi_{n}(\tau, x)+H\left[\psi^{\dagger}, \psi\right]\right],
$$

where $\beta=1 / T$ is the inverse temperature. The limit $T \rightarrow 0$ is to be taken at the end of the calculations.

At this point, it is customary to introduce auxiliary fields for the spin and charge fluctuations via a HubbardStratonpitch (HS) transformation. However, as noted earlier26.27 in the context of systems described by the isotropic 2D Hubbard model, such a procedure immediately leads to loss of spin rotational invariance. The reason for this is that in writing down the Hamiltonian, we have made a particular choice $(\hat{z})$ for the spinquantization axis of the electrons. Other decompositions of $H_{I}$ in terms of charge and spin fluctuations are possible. They are all equivalent as far as the partition function is calculated exactly. The reason for choosing the decomposition (2.4) is that it allows to recover the Hartree-Fock solution at the saddle point level within the functional integral formalism (see Sec. II A).

In order to maintain spin rotational invariance, one should consider the spin-quantization axis to be a priori arbitrary and integrate over all possible directions in the partition function. This is done in practice by introducing a new field $\phi_{n}(\tau, x)$ which is related to the old Fermionic field $\psi_{n}(\tau, x)$ through an unitary $\mathrm{SU}(2) / \mathrm{U}(1)$ rotation matrix $R_{n}(\tau, x)$, i.e. $\psi=R \phi$. The rotation matrix $R$ satisfies $R \sigma_{z} R^{\dagger}=\sigma \cdot \mathbf{n}$, where $\mathbf{n}$ is an unit vector field which gives the direction of spin-quantization axis at point $(x, n)$ and time $\tau$. The new field $\phi$ has its spin-quantization axis along the local $\mathbf{n}$ vector. The interaction term in the action, $S_{I}$ [Eqs. (2.4) and (2.5)], is invariant under this transformation while the unperturbed part, $S_{0}$, becomes

$$
\begin{aligned}
S_{0}= & \sum_{n} \int_{0}^{\beta} d \tau d x\left\{\phi _ { n } ^ { \dagger } ( \tau , x ) \left[\partial_{\tau}-C_{0}\right.\right. \\
& \left.+v_{F}\left(-i \partial_{x} \tau_{3}-k_{F}\right)-v_{F} \tau_{3} C_{x}\right] \phi_{n}(\tau, x) \\
& \left.-t_{b} \sum_{\delta= \pm 1} \phi_{n}^{\dagger}(\tau, x) e^{-i \int_{n b}^{(n+\delta) b} C_{y} d y} \phi_{n+\delta}(\tau, x)\right\} .
\end{aligned}
$$

We have introduced the fields $C_{\mu}$ given by

$$
\begin{aligned}
C_{0} & =-R^{\dagger} \partial_{\tau} R, \\
C_{x} & =i R^{\dagger} \partial_{x} R, \\
e^{-i \int_{n b}^{(n+\delta) b} C_{y} d y} & =R_{n}^{\dagger} R_{n+\delta} .
\end{aligned}
$$

The fields $C_{\mu}$ are $\mathrm{SU}(2)$ gauge fields and the $\mathrm{U}(1)$ gauge freedom here corresponds to an arbitrary rotation about the $z$ axis, which does not change the state of the system. The partition function now contains an additional integral over all $C_{\mu}$ or equivalently $\mathbf{n}$ field configurations. The $C_{\mu}$ fields thus contain information about the spin excitations of the system. In writing Eqs. (2.6) and (2.7), we have considered only long-wavelength fluctuations of the spin-quantization axis, so that the matrix $R$ acts like the unit matrix with respect to the left/right indices of the Fermionic fields.

The $2 k_{F}$ charge fluctuations play no role in the SDW phase, so that we ignore the term $\propto \rho_{c+n}^{*} \rho_{c+n}$ in the interaction Hamiltonian $H_{I}$. We thus write the interaction term in the action as

$$
S_{I}=\frac{U}{4} \sum_{n} \int_{0}^{\beta} d \tau d x\left[\left(\rho_{c n}(\tau, x)\right)^{2}-2 \rho_{s+}^{*}(\tau, x) \rho_{s+}(\tau, x)\right] .
$$

Note that we have kept explicitly the long-wavelength charge fluctuations since they couple to the phason mode 1 In principle, one should also retain the longwavelength spin fluctuations which couple to the spinwave mode. This coupling renormalizes the spin-wave velocity by the usual Stoner factor 1 In practice, it seems difficult within our formalism to treat the longwavelength spin fluctuations in a way that preserves spin rotation invariance. For this reason, we shall ignore them in the following.

We introduce two HS fields, $\rho^{\mathrm{HS}}$ (real) and $\Delta$ (complex), corresponding to charge and spin density fluctuations, respectively. The action of the system can then be written as $S=S_{0}+S_{I}$, where $S_{0}$ is given by Eq. (2.6), and $S_{I}$ by 


$$
\begin{aligned}
S_{I}= & \sum_{n} \int_{0}^{\beta} d \tau d x\left\{-i \rho_{c n}(\tau, x) \rho_{n}^{\mathrm{HS}}(\tau, x)\right. \\
& -\left[\Delta_{n}^{*}(\tau, x) \rho_{s+n}(\tau, x)+\text { c.c. }\right] \\
& \left.+\frac{1}{U}\left[\left(\rho_{n}^{\mathrm{HS}}(\tau, x)\right)^{2}+2\left|\Delta_{n}(\tau, x)\right|^{2}\right]\right\} .
\end{aligned}
$$

Note that the HS field $\rho^{\mathrm{HS}}$ introduced here is not the physical charge-density field, but its conjugate. This can be easily checked by varying the action $S$ with respect to $\rho^{\text {HS }}$. Following Palo et al. 30 we now introduce the physical charge density field $\rho$ by decoupling the quadratic term in $\rho_{n}^{\mathrm{HS}}(\tau, x)$ by means of a HS transformation. This leads to the action

$$
\begin{aligned}
S_{I}= & \sum_{n} \int_{0}^{\beta} d \tau d x\left[-i \rho_{c n}(\tau, x) \rho_{n}^{\mathrm{HS}}(\tau, x)\right. \\
& -\left[\Delta_{n}^{*}(\tau, x) \rho_{s+n}(\tau, x)+\text { c.c. }\right]+\frac{U}{4}\left(\rho_{n}(\tau, x)\right)^{2} \\
& \left.+\frac{2}{U}\left|\Delta_{n}(\tau, x)\right|^{2}+i \rho_{n}^{\mathrm{HS}}(\tau, x) \rho_{n}(\tau, x)\right]
\end{aligned}
$$

It can be seen, by varying the action with respect to the HS field $\rho^{\mathrm{HS}}$, that $\rho$ is indeed the physical charge density field.

Finally, we introduce a weak external electromagnetic field $A_{\mu}$ in the action in a gauge-invariant manner. This does not change $S_{I}$ while $S_{0}$ becomes

$$
\begin{aligned}
S_{0}= & \sum_{n} \int_{0}^{\beta} d \tau d x\left\{\phi _ { n } ^ { \dagger } ( \tau , x ) \left[\partial_{\tau}-e A_{0}-C_{0}\right.\right. \\
& \left.+v_{F}\left(\left(-i \partial_{x}-e A_{x}-C_{x}\right) \tau_{3}-k_{F}\right)\right] \phi_{n}(\tau, x) \\
& \left.-t_{b} \sum_{\delta= \pm 1} \phi_{n}^{\dagger}(\tau, x) e^{-i \int_{n b}^{(n+\delta) b}\left(C_{y}+e A_{y}\right) d y} \phi_{n+\delta}(\tau, x)\right\}
\end{aligned}
$$

Note that, as an artifact of the linearization of the dispersion relation along the chain, $S_{0}$ does not contain the diamagnetic term $\propto A_{x}^{2}$. However, as we shall see, this term is important for recovering a gauge-invariant effective action. We shall recover this term starting from the original tight-binding Hamiltonian in Sec. IIB.

\section{A. Mean-field theory}

The standard mean-field theory is recovered from a saddle-point approximation with $\mathbf{n}=\hat{z}, C_{\mu}=A_{\mu}=0$, $\rho=\rho_{0}, \rho^{\mathrm{HS}}=\rho_{0}^{\mathrm{HS}}$ and $\Delta=\Delta_{0} \exp (i \mathbf{Q} \cdot \mathbf{r})$, where $\mathbf{Q}$ is the nesting wave-vector and $\mathbf{r}=(x, n b)$. We take $\Delta_{0}$ to be real, without any loss of generality. Varying the action with respect to the auxiliary fields $\rho, \rho^{\mathrm{HS}}$ and $\Delta$, we obtain the mean-field equations

$$
\begin{gathered}
\rho_{0}=-\frac{2 i}{U} \rho_{0}^{\mathrm{HS}}=\left\langle\phi_{n}^{\dagger}(\tau, x) \phi_{n}(\tau, x)\right\rangle_{\mathrm{MF}}, \\
\Delta_{0}=\frac{U}{2} e^{-i \mathbf{Q} \cdot \mathbf{r}}\left\langle\phi_{n}^{\dagger}(\tau, x) \tau_{-} \sigma_{z} \phi_{n}(\tau, x)\right\rangle_{\mathrm{MF}} .
\end{gathered}
$$

It is clear from the above equations that the mean field $\rho_{0}$ is the density of particles in the system and has the same value in both the metallic and the SDW ground state. As a result, $-i \rho_{0}^{\mathrm{HS}}$ can be absorbed as a trivial shift in the chemical potential of the system [see Eq. (2.10)]. The equation for $\Delta_{0}$ is the usual mean-field equation for the SDW order parameter.

The mean-field propagator can now be obtained from the mean-field Fermionic action

$$
S_{\mathrm{MF}}=-\frac{1}{\beta} \sum_{\omega_{n}} \sum_{\mathbf{k}, \sigma}\left(\phi_{+\sigma}^{\dagger}\left(i \omega_{n}, \mathbf{k}+\mathbf{Q}\right), \phi_{-\sigma}^{\dagger}\left(i \omega_{n}, \mathbf{k}\right)\right) \mathcal{G}_{\sigma}^{-1}\left(\begin{array}{c}
\phi_{+\sigma}\left(i \omega_{n}, \mathbf{k}+\mathbf{Q}\right) \\
\phi_{-\sigma}\left(i \omega_{n}, \mathbf{k}\right)
\end{array}\right)
$$

where we have dropped additive contributions to the free energy due to the mean-fields $\rho_{0}, \rho_{0}^{\mathrm{HS}}$ and $\Delta_{0}$. Here $\mathcal{G}_{\sigma}^{-1}$ is the inverse propagator given by

$$
\mathcal{G}_{\sigma}^{-1}=\left(\begin{array}{cc}
i \omega_{n}-\epsilon_{+}(\mathbf{k}+\mathbf{Q}) & \operatorname{sgn}(\sigma) \Delta_{0} \\
\operatorname{sgn}(\sigma) \Delta_{0} & i \omega_{n}-\epsilon_{-}(\mathbf{k})
\end{array}\right)
$$

where $\operatorname{sgn}(\sigma)=+(-)$ for $\sigma=\uparrow(\downarrow)$ and $\epsilon_{ \pm}(\mathbf{k})=v_{F}\left( \pm k_{x}-\right.$ $\left.k_{F}\right)-2 t_{b} \cos \left(k_{y} b\right)$ is the dispersion relation for the right and left Fermions.

The mean-field propagator is obtained by inverting $\mathcal{G}_{\sigma}^{-1}$. It is given by

$$
\mathcal{G}_{\sigma}=\left(\begin{array}{cc}
G_{+\sigma}\left(i \omega_{n}, \mathbf{k}+\mathbf{Q}\right) & F_{+\sigma}\left(i \omega_{n}, \mathbf{k}+\mathbf{Q}\right) \\
F_{-\sigma}\left(i \omega_{n}, \mathbf{k}\right) & G_{-\sigma}\left(i \omega_{n}, \mathbf{k}\right)
\end{array}\right)
$$

where

$$
G_{ \pm \sigma}\left(i \omega_{n}, \mathbf{k}\right)=-\left\langle\phi_{ \pm \sigma}\left(i \omega_{n}, \mathbf{k}\right) \phi_{ \pm \sigma}^{\dagger}\left(i \omega_{n}, \mathbf{k}\right)\right\rangle
$$

$$
\begin{aligned}
& =-\frac{i \omega_{n}+\epsilon_{ \pm}(\mathbf{k})}{\omega_{n}^{2}+\epsilon_{ \pm}^{2}+\Delta_{0}^{2}} \\
F_{ \pm \sigma}\left(i \omega_{n}, \mathbf{k}\right) & =-\left\langle\phi_{ \pm \sigma}\left(i \omega_{n}, \mathbf{k}\right) \phi_{\mp \sigma}^{\dagger}\left(i \omega_{n}, \mathbf{k} \mp \mathbf{Q}\right)\right\rangle \\
& =\frac{\operatorname{sgn}(\sigma) \Delta_{0}}{\omega_{n}^{2}+\epsilon_{ \pm}^{2}+\Delta_{0}^{2}}
\end{aligned}
$$

and we have used the relation $\epsilon_{+}(\mathbf{k}+\mathbf{Q})=-\epsilon_{-}(\mathbf{k})$ in obtaining the above result.

\section{B. Fluctuations}

We do not consider amplitude fluctuations, since they are gapped and decouple from the sliding and spin-wave modes in the long-wavelength limit.1 We therefore write the auxiliary fields as 


$$
\begin{aligned}
\rho^{\mathrm{HS}} & =\rho_{0}^{\mathrm{HS}}+\delta \rho^{\mathrm{HS}}, \\
\rho & =\rho_{0}+\delta \rho, \\
\Delta & =\Delta_{0} e^{i(\mathbf{Q} \cdot \mathbf{r}+\theta)},
\end{aligned}
$$

where $\delta \rho, \delta \rho^{\mathrm{HS}}$, and $\theta$ represent small fluctuations of the fields about their mean-field values. The action can then be written as $S=S_{0}^{\prime}+S_{I}^{\prime}$, with

$$
\begin{aligned}
S_{0}^{\prime}= & \sum_{n} \int_{0}^{\beta} d \tau d x\left\{\phi _ { n } ^ { \dagger } ( \tau , x ) \left[\partial_{\tau}-e A_{0}-C_{0}-i \delta \rho^{\mathrm{HS}}\right.\right. \\
& +v_{F}\left(\left(-i \partial_{x}-e A_{x}-C_{x}\right) \tau_{3}-k_{F}\right) \\
& \left.-\Delta_{0}\left(e^{-i(\mathbf{Q} \cdot \mathbf{r}+\theta)} \tau_{-} \sigma_{z}+\text { c.c. }\right)\right] \phi_{n}(\tau, x) \\
& \left.-t_{b} \sum_{\delta= \pm 1} \phi_{n}^{\dagger}(\tau, x) e^{-i \int_{n b}^{(n+\delta) b}\left(C_{y}+e A_{y}\right) d y} \phi_{n+\delta}(\tau, x)\right\}, \\
S_{I}^{\prime}= & \sum_{n} \int_{0}^{\beta} d \tau d x\left[\frac{U}{4}\left(\delta \rho_{n}(\tau, x)\right)^{2}+i \delta \rho_{n}^{\mathrm{HS}}(\tau, x)\right. \\
& \left.\times\left(\rho_{0}+\delta \rho_{n}(\tau, x)\right)\right] .
\end{aligned}
$$

The next step to obtain the effective action of the auxiliary fields $\delta \rho, \delta \rho^{\mathrm{HS}}$ and $\theta$ is to integrate out the Fermion fields. This is most conveniently done by first introducing a new field $\phi^{\prime}$ related to the field $\phi$ through an unitary chiral transformation:

$$
\phi=U_{\text {chiral }} \phi^{\prime}=e^{i \tau_{3} \theta / 2} \phi^{\prime} .
$$

This transformation leaves the interaction part of the action $S_{I}^{\prime}$ invariant while $S_{0}^{\prime}$ is given by

$$
\begin{aligned}
S_{0}^{\prime}= & \sum_{n} \int_{0}^{\beta} d \tau d x\left\{\phi _ { n } ^ { \dagger } ( \tau , x ) \left[\partial_{\tau}-A_{0}^{\mathrm{tot}}\right.\right. \\
& +v_{F}\left(-i \partial_{x} \tau_{3}-k_{F}-A_{x}^{\mathrm{tot}}\right) \\
& \left.-\Delta_{0}\left(e^{-i \mathbf{Q} \cdot \mathbf{r}} \tau_{-} \sigma_{z}+\text { c.c. }\right)\right] \phi_{n}^{\prime}(\tau, x) \\
& \left.-t_{b} \sum_{\delta= \pm 1} \phi_{n}^{\prime \dagger}(\tau, x) e^{-i \int_{n b}^{(n+\delta) b} A_{y}^{\text {tot }} d y} \phi_{n+\delta}^{\prime}(\tau, x)\right\},
\end{aligned}
$$

where the gauge field $A_{\mu}^{\text {tot }}$ is given by

$$
\begin{aligned}
A_{0}^{\text {tot }} & =e A_{0}-\frac{v_{F}}{2} \partial_{x} \theta+i \delta \rho^{\mathrm{HS}}+C_{0}, \\
A_{x}^{\text {tot }} & =\left(e A_{x}-\frac{i}{2 v_{F}} \partial_{\tau} \theta+C_{x}\right) \tau_{3}, \\
A_{y}^{\text {tot }} & =e A_{y}-\frac{1}{2} \partial_{y} \theta \tau_{3}+C_{y} .
\end{aligned}
$$

The chiral transformation therefore eliminates the phase $\theta$ of the order parameter $\Delta$. The action $S_{0}^{\prime}$ [Eq. (2.20)] therefore acquires a simple form. The tradeoff, however, is that the Fermions are now subjected to an effective potential $A_{\mu}^{\text {tot }}$ which contains the derivatives of the phase field $\theta$. Notice that in contrast to the superconducting case, the gradients of the phase couple to the external electromagnetic field in a different manner. This is a consequence of the different broken symmetries in the two cases. Since we assume that the fluctuations of the order parameter (we have taken $\theta_{\mathrm{MF}}=0$ ) are small, the gradients of $\theta$ must be small. As a result, one can carry out a perturbative expansion of the action in $\partial_{\mu} \theta$ or equivalently in $A_{\mu}^{\text {tot }}$. The chiral transformation also produces a non-trivial Jacobian $J$, which yields the additional contribution $S_{J}=-\ln J$ to the action. We shall come back to the origin of this non trivial Jacobian and the method of its calculation in more detail in Sec. II B 2.

We are now in a position to write the action in terms of the mean-field action $S_{\mathrm{MF}}$ [Eq. (2.13)] and the part involving fluctuations. The action then reads $S=S_{0}^{\prime}+S_{I}^{\prime}+S_{J}$, where

$$
\begin{aligned}
S_{0}^{\prime}= & S_{\mathrm{MF}} \\
& -\sum_{n} \int_{0}^{\beta} d \tau d x\left\{\phi_{n}^{\prime \dagger}(\tau, x)\left[A_{0}^{\mathrm{tot}}+v_{F} A_{x}^{\mathrm{tot}}\right] \phi_{n}^{\prime}(\tau, x)\right. \\
& \left.-t_{b} \sum_{\delta= \pm 1} \phi_{n}^{\prime \dagger}(\tau, x)\left(e^{-i \int_{n b}^{(n+\delta) b} A_{y}^{\mathrm{tot}} d y}-1\right) \phi_{n+\delta}^{\prime}(\tau, x)\right\} .
\end{aligned}
$$

Using the fact that $A_{y}^{\text {tot }}$ is a slowly varying weak field, we expand the factor $\exp \left(-i \int_{n b}^{(n+\delta) b} A_{y}^{\text {tot }} d y\right)-1$ in $S_{0}^{\prime}$ in powers of $A_{y}^{\text {tot }}$. The terms in the expansion are written in a symmetric way with respect to $n$ and $n+\delta$. Here, we retain terms up to the quadratic order in $A_{y}^{\text {tot. }}$. The corresponding contribution to the action reads $S_{y}=S_{y}^{\text {linear }}+S_{y}^{\text {dia }}$, where

$$
\begin{aligned}
S_{y}^{\text {linear }}= & \frac{i t_{b} b}{2} \sum_{n, \delta= \pm} \int_{0}^{\beta} d \tau d x \phi_{n}^{\prime \dagger}(\tau, x)\left(A_{y n}^{\mathrm{tot}}(\tau, x)\right. \\
& \left.+A_{y n+\delta}^{\mathrm{tot}}(\tau, x)\right) \delta \phi_{n+\delta}^{\prime}(\tau, x), \\
S_{y}^{\text {dia }}= & \frac{t_{b} b^{2}}{8} \sum_{n, \delta= \pm} \int_{0}^{\beta} d \tau d x \phi_{n}^{\prime \dagger}(\tau, x)\left(A_{y n}^{\mathrm{tot}}(\tau, x)\right. \\
& \left.+A_{y n+\delta}^{\mathrm{tot}}(\tau, x)\right)^{2} \phi_{n+\delta}^{\prime}(\tau, x) .
\end{aligned}
$$

The linear term $S_{y}^{\text {linear }}$ corresponds to the coupling of $A_{y}^{\text {tot }}$ to the paramagnetic current in the transverse direction, while $S_{y}^{\text {dia }}$, which is quadratic in $A_{y}^{\text {tot }}$, is the diamagnetic contribution. If we had started with the original tight-binding Hamiltonian in the $x$ direction, we would have come up, apart from the linear paramagnetic term, with a similar diamagnetic term in the $x$ direction given by

$$
\begin{aligned}
S_{x}^{\text {dia }}= & \frac{t_{a} a^{2}}{2} \sum_{n} \int_{0}^{\beta} d \tau \frac{d k_{x} d p_{x}}{(2 \pi)^{2}} \phi_{n}^{\dagger}\left(k_{x}, \tau\right) \\
& \times\left|e A_{x n}\left(p_{x}, \tau\right)+C_{x n}\left(p_{x}, \tau\right)\right|^{2} \\
& \times\left[\cos \left(k_{x} a\right)+\cos \left(\left(k_{x}-p_{x}\right) a\right)\right] \phi_{n}^{\prime}\left(k_{x}, \tau\right),
\end{aligned}
$$


where $t_{a}$ is the hopping parameter along the chain. $S_{x}^{\text {dia }}$, which is not obtained if one uses a linear dispersion law from the very beginning, has to be included in the action in order to maintain gauge invariance.

It is convenient to introduce the charge and spin currents for the Fermions:

$$
\begin{aligned}
j_{0}^{\alpha} & =\phi^{\prime}{ }^{\dagger} \sigma^{\alpha} \phi^{\prime}, \\
j_{x}^{\alpha} & =\phi^{\prime}{ }^{\dagger} v_{F} \tau_{3} \sigma^{\alpha} \phi^{\prime} \\
j_{y}^{\alpha} & =-\frac{i t_{b} b}{2} \sum_{\delta= \pm 1} \delta\left(\phi_{n-\delta}^{\prime \dagger} \sigma^{\alpha} \phi_{n}^{\prime}+\phi_{n}^{\prime}{ }^{\dagger} \sigma^{\alpha} \phi_{n+\delta}^{\prime}\right), \\
g & =-\frac{i t_{b} b}{2} \sum_{\delta= \pm 1} \delta\left(\phi_{n-\delta}^{\prime \dagger} \tau_{3} \phi_{n}^{\prime}+\phi_{n}^{\prime}{ }^{\dagger} \tau_{3} \phi_{n+\delta}^{\prime}\right),
\end{aligned}
$$

where the index $\alpha$ runs over $0, x, y, z, \sigma^{0}$ is the unit matrix, $j_{\mu}^{0}$ is the $\mu$ th component of the charge current, and $j_{\mu}^{\alpha}$ for $\alpha \neq 0$ give different components of the spin current. The current $g$ is almost same as the charge current in the transverse direction, except that it is chiral, i.e. it has opposite sign for the left and right moving Fermions. This current is introduced for notational convenience, as we shall see later.

Furthermore, since the $C_{\mu}$ fields are $\mathrm{SU}(2)$ gauge fields, it is possible to write them in terms of the $\sigma$ matrices, namely, $C_{\mu}=A_{\mu}^{\nu} \sigma_{\nu}$, where the index $\mu$ runs over $0, x, y$ and the index $\nu$ over $x, y, z$. As a result, the fields $A_{\mu}^{\text {tot }}$ can be expressed as

$$
\begin{aligned}
& A_{0}^{\mathrm{tot}}=A_{0}^{0}+A_{0}^{\nu} \sigma_{\nu}, \\
& A_{x}^{\mathrm{tot}}=\left(A_{x}^{0}+A_{x}^{\nu} \sigma_{\nu}\right) \tau_{3}, \\
& A_{y}^{\mathrm{tot}}=A_{y}^{0}+A_{y}^{\nu} \sigma_{\nu}-\frac{1}{2} \partial_{y} \theta \tau_{3},
\end{aligned}
$$

where the expressions for $A_{\mu}^{0}$ can be deduced from Eqs. (2.21) and (2.26) to be

$$
\begin{aligned}
& A_{0}^{0}=e A_{0}-\frac{v_{F}}{2} \partial_{x} \theta+i \delta \rho^{\mathrm{HS}}, \\
& A_{x}^{0}=e A_{x}-\frac{i}{2 v_{F}} \partial_{\tau} \theta, \\
& A_{y}^{0}=e A_{y} .
\end{aligned}
$$

The action [Eq. (2.22)] can be conveniently expressed in terms of the charge and spin currents as $S=S_{\mathrm{MF}}+S^{\prime \prime}+$ $S^{\text {dia }}+S_{I}^{\prime}+S_{J}$, where

$$
S^{\prime \prime}=-\sum_{n} \int_{0}^{\beta} d \tau d x\left(\sum_{\mu}^{0, x, y, 0, x, y, z} \sum_{\alpha}^{\alpha} A_{\mu}^{\alpha} j_{\mu}-\frac{1}{2} g \partial_{y} \theta\right)
$$

and $S^{\text {dia }}=S_{x}^{\text {dia }}+S_{y}^{\text {dia }}$ is given by Eqs. 2.23) and (2.24).

Integrating out the Fermions, we obtain to quadratic order in the fields $A_{\mu}^{\text {tot }}$ the effective action

$$
S_{\mathrm{eff}}=S_{I}^{\prime}+S_{J}+\left\langle S^{\prime \prime}+S^{\mathrm{dia}}-\frac{S^{\prime \prime 2}}{2}\right\rangle_{\mathrm{MF}}
$$

where $\langle\cdots\rangle_{\mathrm{MF}}$ means that the average is taken with respect to the mean-field action $S_{\mathrm{MF}}$.

The evaluation of $\left\langle S^{\prime \prime}\right\rangle_{\mathrm{MF}}$ is trivial. Only the term involving $j_{0}^{0}$ contributes. We thus obtain

$$
\left\langle S^{\prime \prime}\right\rangle_{\mathrm{MF}}=-\rho_{0} \int_{0}^{\beta} d \tau d^{2} r\left(i \delta \rho^{\mathrm{HS}}+e A_{0}-\frac{v_{F}}{2} \partial_{x} \theta\right) .
$$

Here, we have taken the continuum limit at the end of the calculation and replaced the sum over the chains by an integral in the $y$ direction. It can be easily seen from Eqs. 2.18) and (2.30) that the first term in $\left\langle S^{\prime \prime}\right\rangle_{\mathrm{MF}}$ cancels the term which is linear in $\delta \rho^{\mathrm{HS}}$ in the expression of $S_{I}^{\prime}$. Thus, we finally obtain

$$
\left\langle S^{\prime \prime}\right\rangle_{\mathrm{MF}}=-\rho_{0} \int_{0}^{\beta} d \tau d^{2} r\left(e A_{0}-\frac{v_{F}}{2} \partial_{x} \theta\right)
$$

and

$$
S_{I}^{\prime}=\int_{0}^{\beta} d \tau d^{2} r\left[\frac{U}{4}(\delta \rho)^{2}+i \delta \rho^{\mathrm{HS}} \delta \rho\right]
$$

It may seem unphysical at first sight that the scalar potential in the effective action couples to the constant mean-field density $\rho_{0}$ and not to the full density $\rho=\rho_{0}+\delta \rho$, as one would intuitively expect. However, one should bear in mind that one still has to integrate over $\delta \rho^{\mathrm{HS}}$. As shown by Palo et al 30 in the context of superconducting systems, by redefining the field $\delta \rho^{\mathrm{HS}} \rightarrow \delta \rho^{\mathrm{HS}}-\left(e A_{0}-\frac{v_{F}}{2} \partial_{x} \theta\right)$, we immediately get the coefficient of the scalar potential to be the full density $\rho$ and not $\rho_{0}$.

In the next three sections, we evaluate the diamagnetic term $\left\langle S^{\text {dia }}\right\rangle_{\mathrm{MF}}$, the contribution $S_{J}$ arising from the chiral anomaly, and $\left\langle S^{\prime \prime 2}\right\rangle_{\mathrm{MF}}$.

\section{Diamagnetic contribution}

In this section, we calculate the contribution of the diamagnetic term to the effective action. From Eq. (2.23), one can easily obtain in Fourier space

$$
\left\langle S_{y}^{\text {dia }}\right\rangle_{\mathrm{MF}}=\frac{t_{b} b^{2}}{2} \frac{1}{\beta} \sum_{p_{n}} \int \frac{d^{2} p}{(2 \pi)^{2}}\left(e^{2}\left|A_{y}\left(i p_{n}, \mathbf{p}\right)\right|^{2}+\sum_{\nu}^{x, y, z}\left|A_{y}^{\nu}\left(i p_{n}, \mathbf{p}\right)\right|^{2}+\frac{p_{y}^{2}}{4}\left|\theta\left(i p_{n}, \mathbf{p}\right)\right|^{2}\right)
$$




$$
\times \frac{1}{\beta} \sum_{\alpha= \pm, \sigma, \omega_{n}} \int \frac{d^{2} k}{(2 \pi)^{2}}\left[\cos \left(k_{y} b\right)+\cos \left(\left(k_{y}-p_{y}\right) b\right)\right] G_{\alpha \sigma}\left(i \omega_{n}, \mathbf{k}\right)
$$

where we use $A_{y}^{*}\left(i p_{n}, \mathbf{p}\right)=A_{y}\left(-i p_{n},-\mathbf{p}\right) \ldots$ for real fields and $G_{ \pm \sigma}$ is the mean-field propagator given by $(2.16)$. In the limit $t_{b} \gg \Delta_{0}, 31$ we can ignore the effect of the gap and replace $G_{ \pm \sigma}$ by its value in the metallic phase. To first order in $p_{y}^{2}$, we then have

$$
\begin{aligned}
\frac{1}{\beta} \sum_{\alpha= \pm, \sigma, \omega_{n}} \int \frac{d^{2} k}{(2 \pi)^{2}}\left[\cos \left(k_{y} b\right)+\cos \left(\left(k_{y}-p_{y}\right) b\right)\right] G_{\alpha \sigma}\left(i \omega_{n}, \mathbf{k}\right) & =4 N(0)\left(1-\frac{p_{y}^{2} b^{2}}{4}\right) \int \frac{d k_{y}}{2 \pi} \cos \left(k_{y} b\right) \\
& \times \int_{-2 t_{b}}^{2 t_{b}} d \epsilon \Theta\left(-\epsilon+2 t_{b} \cos \left(k_{y} b\right)\right), \\
& =\frac{2 N(0) v_{\perp}^{2}}{t_{b} b^{2}}\left(1-\frac{p_{y}^{2} b^{2}}{4}\right),
\end{aligned}
$$

where $v_{\perp}=\sqrt{2 t_{b}^{2} b^{2}}$ is the velocity of the Fermions in the transverse direction, $N(0)=1 / \pi v_{F} b$ is the density of states per spin, and $\Theta$ is the step function. Note that only the states near the Fermi level $\left(|\epsilon| \leq 2 t_{b}\right)$ contribute to the diamagnetic term $\left\langle S_{y}^{\text {dia }}\right\rangle_{\mathrm{MF}}$, so that the use of a linearized dispersion law is justified. Substituting Eq. (2.34) in Eq. (2.33), we finally get

$$
\left\langle S_{y}^{\text {dia }}\right\rangle_{\mathrm{MF}}=N(0) v_{\perp}^{2} \frac{1}{\beta} \sum_{p_{n}} \int \frac{d^{2} p}{(2 \pi)^{2}}\left(1-\frac{p_{y}^{2} b^{2}}{4}\right)\left(e^{2}\left|A_{y}\left(i p_{n}, \mathbf{p}\right)\right|^{2}+\sum_{\nu}^{x, y, z}\left|A_{y}^{\nu}\left(i p_{n}, \mathbf{p}\right)\right|^{2}+\frac{p_{y}^{2}}{4}\left|\theta\left(i p_{n}, \mathbf{p}\right)\right|^{2}\right) .
$$

The calculation of the diamagnetic term in the $x$ direction should be done using the original tight-binding dispersion law, since it involves electronic states deep in the Fermi sea which forbids the use of a linearized dispersion law. From Eq. (2.24), we deduce

$$
\begin{aligned}
\left\langle S_{x}^{\mathrm{dia}}\right\rangle_{\mathrm{MF}}= & \frac{t_{a} a^{2}}{2} \frac{1}{\beta} \sum_{p_{n}} \int \frac{d^{2} p}{(2 \pi)^{2}}\left(e^{2}\left|A_{x}\left(i p_{n}, \mathbf{p}\right)\right|^{2}+\sum_{\nu}^{x, y, z}\left|A_{x}^{\nu}\left(i p_{n}, \mathbf{p}\right)\right|^{2}\right) \\
& \times \frac{1}{\beta} \sum_{\sigma, \omega_{n}} \int \frac{d^{2} k}{(2 \pi)^{2}}\left[\cos \left(k_{x} a\right)+\cos \left(\left(k_{x}-p_{x}\right) a\right)\right] G_{\sigma}\left(i \omega_{n}, \mathbf{k}\right),
\end{aligned}
$$

where $G_{\sigma}^{-1}\left(i \omega_{n}, \mathbf{k}\right)=i \omega_{n}+2 t_{a} \cos \left(k_{x} a\right)+2 t_{b} \cos \left(k_{y} b\right)+\mu$ ( $\mu$ being the Fermi level). Again, we neglect the effect of the gap $\Delta_{0}$. Neglecting corrections of order $t_{b} / t_{a}$, we have

$\frac{1}{\beta} \sum_{\sigma, \omega_{n}} \int \frac{d^{2} k}{(2 \pi)^{2}}\left[\cos \left(k_{x} a\right)+\cos \left(\left(k_{x}-p_{x}\right) a\right)\right] G_{\sigma}\left(i \omega_{n}, \mathbf{k}\right)=\frac{2}{\pi b} \int_{-\pi / a}^{\pi / a} \frac{d k_{x}}{2 \pi} \cos \left(k_{x} a\right) \Theta\left(2 t_{a} \cos \left(k_{x} a\right)+\mu\right)=v_{F}^{2} N(0)$,

which yields

$$
\left\langle S_{x}^{\text {dia }}\right\rangle_{\mathrm{MF}}=N(0) v_{F}^{2} \frac{1}{\beta} \sum_{p_{n}} \int \frac{d^{2} p}{(2 \pi)^{2}}\left(e^{2}\left|A_{x}\left(i p_{n}, \mathbf{p}\right)\right|^{2}+\sum_{\nu}^{x, y, z}\left|A_{x}^{\nu}\left(i p_{n}, \mathbf{p}\right)\right|^{2}\right) .
$$

We do not consider the term of order $q_{x}^{2}$, which is consistent with the linearized dispersion law used in the rest of the calculation.

\section{Chiral anomaly}

In this section, we calculate the action $S_{J}$ due to the Jacobian of the chiral transformation (2.19). The lat- ter produces not only a change of the gauge fields $A_{\mu}^{\text {tot }}$ [Eqs. (2.26) and (2.27)], but also changes the ground state of the system. This non-perturbative effect shows up in the Jacobian of the chiral transformation. Chiral anomalies have been known for a long time in the context of DW systems. 18 20,23 24 Our method of calculation is similar to that of Ref. 24.

The chiral transformation changes the local density of particle in the ground state (the total particle number re- 
maining unchanged). Since the particle density couples to the gauge field $A_{0}^{0}$, this yields an additional contribution, $S_{J}$, to the action.

Let us first calculate the density change $\delta \rho(\tau, \mathbf{r})$ due to an infinitesimal chiral transformation,

$$
U_{\text {chiral }}[\delta \theta(\tau, \mathbf{r})]=e^{\frac{i}{2} \tau_{3} \delta \theta(\tau, \mathbf{r})}
$$

which changes the phase of the order parameter from $\theta(\tau, \mathbf{r})$ to $\theta(\tau, \mathbf{r})-\delta \theta(\tau, \mathbf{r})$ :

$$
\delta \rho(\tau, \mathbf{r})=\lim _{\delta \mathbf{r}, \delta \tau \rightarrow 0}\left\langle\phi^{\dagger}(\tau+\delta \tau, \mathbf{r}+\delta \mathbf{r})\left(U_{\text {chiral }}^{\dagger}[\delta \theta(\tau+\delta \tau, \mathbf{r}+\delta \mathbf{r})] U_{\text {chiral }}[\delta \theta(\tau, \mathbf{r})]-1\right) \phi(\tau, \mathbf{r})\right\rangle
$$

where wuse the point spitting regularization scheme. A regularization is necessary to properly calculate the particle density.24 To lowest order in $\delta \theta$, we obtain

$$
\begin{aligned}
\delta \rho(\tau, \mathbf{r}) & =-\frac{i}{2} \lim _{\delta \mathbf{r}, \delta \tau \rightarrow 0}[\delta \theta(\tau+\delta \tau, \mathbf{r}+\delta \mathbf{r})-\delta \theta(\tau, \mathbf{r})]\left\langle\phi^{\dagger}(\tau+\delta \tau, \mathbf{r}+\delta \mathbf{r}) \tau_{3} \phi(\tau, \mathbf{r})\right\rangle \\
& =-\frac{i}{2} \lim _{\delta \mathbf{r}, \delta \tau \rightarrow 0}[\delta \theta(\tau+\delta \tau, \mathbf{r}+\delta \mathbf{r})-\delta \theta(\tau, \mathbf{r})] \sum_{\alpha, \sigma} \alpha G_{\alpha \sigma}(-\delta \tau,-\delta \mathbf{r}),
\end{aligned}
$$

where $G_{\alpha \sigma}$ is the mean-field propagator defined in Sec. II A. 33 Introducing the Fourier transforms $\delta \theta\left(i p_{n}, \mathbf{p}\right)$ and $G_{\alpha \sigma}\left(i \omega_{n}, \mathbf{k}\right), \delta \rho$ is rewritten as

$$
\delta \rho(\tau, \mathbf{r})=-\frac{i}{2 \beta^{2}} \sum_{p_{n}, \omega_{n}} \int \frac{d^{2} p}{(2 \pi)^{2}} \int \frac{d^{2} k}{(2 \pi)^{2}} \delta \theta\left(i p_{n}, \mathbf{p}\right) e^{i \mathbf{p} \cdot \mathbf{r}-i p_{n} \tau} \sum_{\alpha, \sigma} \alpha\left[G_{\alpha \sigma}\left(i \omega_{n}+i p_{n}, \mathbf{k}+\mathbf{p}\right)-G_{\alpha \sigma}\left(i \omega_{n}, \mathbf{k}\right)\right],
$$

after a trivial shift of integration variables, and in the limit $\delta \tau=\delta \mathbf{r}=0$. Performing the sum over $\omega_{n}$, we obtain

$$
\delta \rho(\tau, \mathbf{r})=-\frac{i}{\beta} \sum_{p_{n}} \int \frac{d^{2} p}{(2 \pi)^{2}} \int \frac{d^{2} k}{(2 \pi)^{2}} \delta \theta\left(i p_{n}, \mathbf{p}\right) e^{i \mathbf{p} \cdot \mathbf{r}-i p_{n} \tau} \sum_{\alpha} \alpha\left[n_{\alpha}(\mathbf{k}+\mathbf{p})-n_{\alpha}(\mathbf{k})\right]
$$

where $n_{\alpha}(\mathbf{k})=\Theta\left(k_{F}-\alpha k_{x}+\left(2 t_{b} / v_{F}\right) \cos \left(k_{y} b\right)\right)$ is the particle occupation number. [Note that $n_{\alpha}(\mathbf{k})$ has the same value in the metallic and SDW phases at $T=0$.] Using

$$
n_{\alpha}(\mathbf{k}+\mathbf{p})-n_{\alpha}(\mathbf{k})=-\left(\alpha p_{x}+\frac{2 t_{b}}{v_{F}} p_{y} \sin \left(k_{y} b\right)\right) \delta\left(k_{F}-\alpha k_{x}+\frac{2 t_{b}}{v_{F}} \cos \left(k_{y} b\right)\right)
$$

we finally obtain, to lowest order in $\mathbf{p}$,

$$
\begin{aligned}
\delta \rho(\tau, \mathbf{r}) & =\frac{i}{\pi b \beta} \sum_{p_{n}} \int \frac{d^{2} p}{(2 \pi)^{2}} p_{x} \delta \theta\left(i p_{n}, \mathbf{p}\right) e^{i \mathbf{p} \cdot \mathbf{r}-i p_{n} \tau} \\
& =\frac{1}{\pi b} \partial_{x} \delta \theta(\tau, \mathbf{r})
\end{aligned}
$$

By integrating out the Fermions, we have shown that the ground-state density of Fermions couples to the gauge field $A_{0}^{0}$ [Eq. (2.30)]. Since an infinitesimal chiral transformation changes the density by $\delta$ it also produces the contribution to the effective action 34

$$
\begin{aligned}
\delta S_{J} & =-\int_{0}^{\beta} d^{2} r d \tau \delta \rho A_{0}^{0} \\
& =-\frac{1}{\pi b} \int_{0}^{\beta} d^{2} r d \tau \partial_{x} \delta \theta\left(e A_{0}+i \delta \rho^{\mathrm{HS}}-\frac{v_{F}}{2} \partial_{x} \theta\right) .
\end{aligned}
$$

Taking a variational integral of Eq. (2.46), we obtain the chiral anomaly contribution to the effective action

$$
\begin{aligned}
S_{J}= & -v_{F} N(0) \int_{0}^{\beta} d^{2} r d \tau\left[\partial_{x} \theta\left(e A_{0}+i \delta \rho^{\mathrm{HS}}\right)\right. \\
& \left.-\frac{v_{F}}{4}\left(\partial_{x} \theta\right)^{2}\right],
\end{aligned}
$$

where we have used $1 / \pi b=v_{F} N(0)$.

In deriving the action $S_{J}$, we have implicitly assumed that the chiral transformation only produces a change in the density of the ground state. Following analogous steps leading to Eq. (2.45), it can be shown that the chiral transformation does not change the spin density and the spin or charge currents, so that the change in the density is the only non-perturbative effect of the chiral transformation.

\section{3. $\left\langle S^{\prime \prime 2}\right\rangle_{\mathrm{MF}}$}

In this section, we calculate $\left\langle S^{\prime \prime 2}\right\rangle_{\mathrm{MF}}$. Using (2.28), we deduce 


$$
\begin{aligned}
\left\langle S^{\prime \prime 2}\right\rangle_{\mathrm{MF}}= & \frac{1}{\beta} \sum_{p_{n}} \int \frac{d^{2} p}{(2 \pi)^{2}}\left[\sum_{\alpha \beta}^{0, x, y, z} \sum_{\mu \nu}^{0, x, y} A_{\mu}^{\alpha}\left(i p_{n}, \mathbf{p}\right) \Pi_{j_{\mu}^{\alpha} j_{\nu}^{\beta}}\left(i p_{n}, \mathbf{p}\right) A_{\nu}^{\beta}\left(-i p_{n},-\mathbf{p}\right)\right. \\
& +\sum_{\alpha}^{0, x, y, z} \sum_{\mu}^{0, x, y} A_{\mu}^{\alpha}\left(i p_{n}, \mathbf{p}\right) \Pi_{j_{\mu}^{\alpha} g}\left(i p_{n}, \mathbf{p}\right) i p_{y} \theta\left(-i p_{n},-\mathbf{p}\right)+\frac{p_{y}^{2}}{4}\left|\theta\left(i p_{n}, \mathbf{p}\right)\right|^{2} \Pi_{g g}\left(i p_{n}, \mathbf{p}\right),
\end{aligned}
$$

where $\Pi_{j_{\mu}^{\alpha} j_{\nu}^{\beta}}\left(i p_{n}, \mathbf{p}\right), \Pi_{j_{\mu}^{\alpha} g}\left(i p_{n}, \mathbf{p}\right)$ and $\Pi_{g g}\left(i p_{n}, \mathbf{p}\right)$ are the current-current correlators defined by

$$
\begin{aligned}
\Pi_{j_{\mu}^{\alpha} j_{\nu}^{\beta}}\left(i p_{n}, \mathbf{p}\right) & =\left\langle j_{\mu}^{\alpha}\left(i p_{n}, \mathbf{p}\right) j_{\nu}^{\beta}\left(-i p_{n},-\mathbf{p}\right)\right\rangle_{\mathrm{MF}} \\
\Pi_{j_{\mu}^{\alpha}} g & \left(i p_{n}, \mathbf{p}\right)=\left\langle j_{\mu}^{\alpha}\left(i p_{n}, \mathbf{p}\right) g\left(-i p_{n},-\mathbf{p}\right)\right\rangle_{\mathrm{MF}} \\
\Pi_{g g}\left(i p_{n}, \mathbf{p}\right) & =\left\langle g\left(i p_{n}, \mathbf{p}\right) g\left(-i p_{n},-\mathbf{p}\right)\right\rangle_{\mathrm{MF}}
\end{aligned}
$$

and the currents $j_{\mu}^{\alpha}\left(i p_{n}, \mathbf{p}\right)$ and $g\left(i p_{n}, \mathbf{p}\right)$ are the Fourier transforms of the charge, spin and chiral currents defined in Eq. (2.25).

In the long-wavelength limit, we need to compute the effective action to second order in a gradient expansion. For symmetry reasons, the correlators $\Pi_{j_{\mu}^{x, y} j_{\mu^{\prime}}^{0, z}}, \Pi_{j_{\mu}^{x, y} g}$, $\Pi_{j_{\mu}^{x} j_{\mu^{\prime}}^{y}}$, and $\Pi_{j_{\mu}^{z} g}$ vanish, and $\Pi_{j_{\mu}^{x} j_{\mu^{\prime}}^{x}}=\Pi_{j_{\mu}^{y} j_{\mu^{\prime}}^{y}}$. We also find that none of the spin currents $j_{\mu}^{x, y, z}$ couple to the charge currents $j_{\mu}^{0}$ or $g$, which simply states that the sliding and spin-wave modes decouple in the long-wavelength limit. Since the $\mathrm{SU}(2)$ gauge fields are first order in gradient $\left(A_{\mu}^{\nu} \propto \partial_{\mu} n^{\nu}\right)$, we need $\Pi_{j_{\mu}^{\nu} j_{\mu^{\prime}}}$ to zeroth order. This also holds for the correlator $\Pi_{g g}$, since it couples to $\left(\partial_{y} \theta\right)^{2}$. Based on similar reasoning, we must obtain $\Pi_{g j_{\mu}^{0}}$ to first order and $\Pi_{j_{\mu}^{0} j_{\mu^{\prime}}^{0}}$ to second order. As shown in the Appendix, we thus obtain

$$
\begin{aligned}
& \Pi_{j_{0}^{0} j_{0}^{0}}\left(i p_{n}, \mathbf{p}\right)=N(0) \frac{v_{F}^{2} p_{x}^{2}+v_{\perp}^{2} p_{y}^{2}}{3 \Delta_{0}^{2}}, \\
& \Pi_{j_{x}^{0} j_{x}^{0}}\left(i p_{n}, \mathbf{p}\right)=2 N(0) v_{F}^{2}\left(1-\frac{p_{n}^{2}}{6 \Delta_{0}^{2}}\right), \\
& \Pi_{j_{y}^{0} j_{y}^{0}}\left(i p_{n}, \mathbf{p}\right)=2 N(0) v_{\perp}^{2}\left(1-\frac{p_{y}^{2} b^{2}}{4}-\frac{p_{n}^{2}}{6 \Delta_{0}^{2}}\right), \\
& \Pi_{j_{0}^{0} j_{x}^{0}}\left(i p_{n}, \mathbf{p}\right)=N(0) v_{F}^{2} \frac{i p_{n} p_{x}}{3 \Delta_{0}^{2}}, \\
& \Pi_{j_{0}^{0} j_{y}^{0}}\left(i p_{n}, \mathbf{p}\right)=N(0) v_{\perp}^{2} \frac{i p_{n} p_{y}}{3 \Delta_{0}^{2}}, \\
& \Pi_{j_{0}^{x} j_{0}^{x}}\left(i p_{n}, \mathbf{p}\right)=\Pi_{j_{0}^{y} j_{0}^{y}}\left(i p_{n}, \mathbf{p}\right)=2 N(0), \\
& \Pi_{j_{x}^{z} j z}\left(i p_{n}, \mathbf{p}\right)=2 N(0) v_{F}^{2}, \\
& \Pi_{j_{y}^{z} j z}^{z}\left(i p_{n}, \mathbf{p}\right)=2 N(0) v_{\perp}^{2} .
\end{aligned}
$$

Separating charge and spin contributions, we have $-\left\langle S^{\prime \prime 2}\right\rangle_{\mathrm{MF}} / 2=S_{2 \mathrm{spin}}^{\mathrm{eff}}+S_{2 \text { phason }}^{\mathrm{eff}}$, where

$$
\begin{aligned}
S_{2 \text { spin }}^{\text {eff }}= & -N(0) \frac{1}{\beta} \sum_{p_{n}} \int \frac{d^{2} p}{(2 \pi)^{2}}\left[\left|A_{0}^{x}\left(i p_{n}, \mathbf{p}\right)\right|^{2}+\left|A_{0}^{y}\left(i p_{n}, \mathbf{p}\right)\right|^{2}+v_{F}^{2}\left|A_{x}^{z}\left(i p_{n}, \mathbf{p}\right)\right|^{2}+v_{\perp}^{2}\left|A_{y}^{z}\left(i p_{n}, \mathbf{p}\right)\right|^{2}\right], \\
S_{2 \text { phason }}^{\text {eff }}= & -N(0) \frac{1}{\beta} \sum_{p_{n}} \int \frac{d^{2} p}{(2 \pi)^{2}}\left\{\left|e A_{0}\left(i p_{n}, \mathbf{p}\right)+i \delta \rho^{\mathrm{HS}}\left(i p_{n}, \mathbf{p}\right)\right|^{2}\left(\frac{v_{F}^{2} p_{x}^{2}+v_{\perp}^{2} p_{y}^{2}}{6 \Delta_{0}^{2}}\right)+e^{2}\left|A_{x}\left(i p_{n}, \mathbf{p}\right)\right|^{2} v_{F}^{2}\left(1-\frac{p_{n}^{2}}{6 \Delta_{0}^{2}}\right)\right. \\
& +e^{2}\left|A_{y}\left(i p_{n}, \mathbf{p}\right)\right|^{2} v_{\perp}^{2}\left(1-\frac{p_{y}^{2} b^{2}}{4}-\frac{p_{n}^{2}}{6 \Delta_{0}^{2}}\right)-\frac{1}{4}\left|\theta\left(i p_{n}, \mathbf{p}\right)\right|^{2} p_{n}^{2}-\theta\left(i p_{n}, \mathbf{p}\right) e v_{F} p_{n} A_{x}\left(-i p_{n},-\mathbf{p}\right) \\
& \left.+\frac{i p_{n}}{3 \Delta_{0}^{2}}\left[e A_{0}\left(i p_{n}, \mathbf{p}\right)+i \delta \rho^{\mathrm{HS}}\left(i p_{n}, \mathbf{p}\right)\right]\left[v_{F}^{2} p_{x} e A_{x}\left(-i p_{n},-\mathbf{p}\right)+v_{\perp}^{2} p_{y} e A_{y}\left(-i p_{n},-\mathbf{p}\right)\right]\right\} .
\end{aligned}
$$

This completes our derivation of the effective action. In the next sections, we shall use this effective action to obtain the spin-wave and the phason modes.

\section{SPIN-WAVE MODE}

\section{A. $\mathbf{N L} \sigma \mathrm{M}$}

The contribution to the spin part $S_{\mathrm{spin}}^{\mathrm{eff}}$ of the effective action comes from the terms involving the gauge fields $A_{\mu}^{x, y, z}$. From Eqs. (2.35), 2.38) and (2.51), we find

$$
\begin{aligned}
S_{\mathrm{spin}}^{\mathrm{eff}}= & -N(0) \sum_{\nu}^{x, y} \frac{1}{\beta} \sum_{p_{n}} \int \frac{d^{2} p}{(2 \pi)^{2}}\left[\left|A_{0}^{\nu}\left(i p_{n}, \mathbf{p}\right)\right|^{2}\right. \\
& \left.-v_{F}^{2}\left|A_{x}^{\nu}\left(i p_{n}, \mathbf{p}\right)\right|^{2}-v_{\perp}^{2}\left|A_{y}^{\nu}\left(i p_{n}, \mathbf{p}\right)\right|^{2}\right] .
\end{aligned}
$$


The contribution from the diamagnetic terms [Eqs. 2.35) and (2.38)] cancels the terms proportional to $A_{\mu}^{z}$ in Eq. (2.51), so that we are finally left with an effective action which depends only on $A_{\mu}^{x}$ and $A_{\mu}^{y}$. This cancelation is expected since our effective action has to be gauge invariant. As mentioned earlier, for the $\mathrm{SU}(2)$ fields $C_{\mu}$ a gauge transformation corresponds to an arbitrary rotation about the $z$ axis, and does not change the state of the system. Such a rotation changes $A_{\mu}^{z} \rightarrow A_{\mu}^{z}+1 / 2 \partial_{\mu} \Lambda$, where $\Lambda$ is the rotation angle.35 Thus to be gauge-invariant, the effective action cannot depend on the $A_{\mu}^{z}$ field. The diamagnetic terms in the effective action are absolutely crucial for this cancelation and hence for obtaining a gauge-invariant effective action for the spin-wave mode

Using the identities 35

$$
\begin{aligned}
\operatorname{tr}\left(C_{(x, y)}^{2}\right)-\operatorname{tr}\left(\sigma_{z} C_{(x, y)}\right)^{2} & =A_{(x, y)}^{x}{ }^{2}+A_{(x, y)}^{y}{ }^{2} \\
& =\frac{\left(\partial_{(x, y)} \mathbf{n}\right)^{2}}{4}, \\
\operatorname{tr}\left(C_{0}^{2}\right)-\operatorname{tr}\left(\sigma_{z} C_{0}\right)^{2} & =A_{0}^{x 2}+A_{0}^{y 2}=-\frac{\left(\partial_{\tau} \mathbf{n}\right)^{2}}{4},
\end{aligned}
$$

we express the effective action [Eq. (3.1)] in terms of gradients of the $\mathbf{n}$ field:

$S_{\mathrm{spin}}^{\mathrm{eff}}=\frac{N(0)}{4} \int_{0}^{\beta} d^{2} r d \tau\left[\left(\partial_{\tau} \mathbf{n}\right)^{2}+v_{F}^{2}\left(\partial_{x} \mathbf{n}\right)^{2}+v_{\perp}^{2}\left(\partial_{y} \mathbf{n}\right)^{2}\right]$.

The effective action for the spins therefore turns out to be an anisotropic NL $\sigma \mathrm{M}$, as expected. Since $S_{\text {spin }}^{\text {eff }}$ has been obtained within a gradient expansion, valid for $\left|p_{n}\right|, v_{F}\left|p_{x}\right|, v_{\perp}\left|p_{y}\right| \ll \Delta_{0}$, the NL $\sigma \mathrm{M}$ describes the behavior of the model only in the low-energy longwavelength limit. Thus, Eq. (3.3) should be supplemented with cut-offs $\Lambda_{0} \sim \Delta_{0}$ in energy space and $\Lambda_{x} \sim \Delta_{0} / v_{F}$ and $\Lambda_{y} \sim \Delta_{0} / v_{\perp}$ in momentum space.

The action (3.3) can be expressed in a more conventional form 3 as

$$
S_{\mathrm{spin}}^{\mathrm{eff}}=\frac{1}{2} \int_{0}^{\beta} d^{2} r d \tau\left[\chi\left(\partial_{\tau} \mathbf{n}\right)^{2}+\rho_{s x}\left(\partial_{x} \mathbf{n}\right)^{2}+\rho_{s y}\left(\partial_{y} \mathbf{n}\right)^{2}\right],
$$

where we have introduced the parameters $\chi=N(0) / 2$, $\rho_{s x}=N(0) v_{F}^{2} / 2$ and $\rho_{s y}=N(0) v_{\perp}^{2} / 2$. Note that these values are only approximate. In order to obtain the correct low-energy long-wavelength behavior, it would be necessary to integrate out all high-energy short-wavelength fluctuations. The latter are expected to renormalize the bare values of $\chi, \rho_{s x}$ and $\rho_{s y}$ quoted above.

To see the physical interpretation of the parameter $\chi$, we note that $\chi=\Pi_{j_{0}^{\nu} j_{0}^{\nu}}\left(i p_{n}=0, \mathbf{p}=0\right) / 4(\nu=$ $x, y)$. The correlators $\Pi_{j_{0}^{x} j_{0}^{x}}^{x}$ and $\Pi_{j_{0}^{y} j_{0}^{y}}$ can be linked to the transverse spin susceptibilities in the mean-field state, i.e. $\Pi_{j_{0}^{x(y)} j_{0}^{x(y)}}=4\left\langle S_{x(y)} S_{x(y)}\right\rangle_{\mathrm{MF}}$, where $S_{\mu}=$ $\phi^{\dagger} \sigma_{\mu} \phi / 2$. We see that $\chi=\left\langle S_{x} S_{x}\right\rangle_{\mathrm{MF}}=\left\langle S_{y} S_{y}\right\rangle_{\mathrm{MF}}$ and hence corresponds to the transverse static uniform spin-susceptibility of the system in the mean-field ground state.

The spin stiffness coefficients $\rho_{s x}$ and $\rho_{s y}$, on the other hand, come from the diamagnetic terms [Eqs. 2.35) and (2.38)], which are themselves related to the average kinetic energy. To see this point more clearly, let us consider the diamagnetic term in the $y$ direction. We have

$$
\begin{aligned}
N(0) v_{\perp}^{2} \sum_{\mu}^{x, y} \frac{1}{\beta} \sum_{p_{n}} \int \frac{d^{2} p}{(2 \pi)^{2}}\left|A_{y}^{\mu}\left(i p_{n}, \mathbf{p}\right)\right|^{2} & =\frac{t_{b} b^{2}}{2} \sum_{\mu}^{x, y} \frac{1}{\beta} \sum_{p_{n}} \int \frac{d^{2} p}{(2 \pi)^{2}}\left|A_{y}^{\mu}\left(i p_{n}, \mathbf{p}\right)\right|^{2} \frac{1}{\beta} \sum_{i \omega_{n}, \alpha, \sigma} \frac{d^{2} k}{(2 \pi)^{2}} 2 \cos \left(k_{y} b\right) G_{\alpha \sigma}\left(i \omega_{n}, \mathbf{k}\right) \\
& =-\frac{b^{2}}{2}\left\langle K_{y}\right\rangle_{\mathrm{MF}} \sum_{\mu}^{x, y} \frac{1}{\beta} \sum_{p_{n}} \int \frac{d^{2} p}{(2 \pi)^{2}}\left|A_{y}^{\mu}\left(i p_{n}, \mathbf{p}\right)\right|^{2},
\end{aligned}
$$

where $\left\langle K_{y}\right\rangle_{\mathrm{MF}}$ is the average kinetic energy in the $y$ direction per unit area in the mean-field state. But we can also write the diamagnetic term as

$$
N(0) v_{\perp}^{2} \sum_{\mu}^{x, y} \frac{1}{\beta} \sum_{p_{n}} \int \frac{d^{2} p}{(2 \pi)^{2}}\left|A_{y}^{\mu}\left(i p_{n}, \mathbf{p}\right)\right|^{2}=\frac{\rho_{s y}}{2} \int_{0}^{\beta} d \tau d^{2} r\left(\frac{\partial \mathbf{n}}{\partial y}\right)^{2} .
$$

Comparing Eqs. (3.5) and (3.6), we immediately see that the spin stiffness parameter $\rho_{s y}$ is related to the average kinetic energy in the $y$ direction. A similar result can be obtained for $\rho_{s x}$, and we finally get

$$
\rho_{s x}=-\frac{a^{2}\left\langle K_{x}\right\rangle_{\mathrm{MF}}}{4}
$$

$$
\rho_{s y}=-\frac{b^{2}\left\langle K_{y}\right\rangle_{\mathrm{MF}}}{4} .
$$

The velocities of the spin-wave mode, $v_{x}=\left(\rho_{s x} / \chi\right)^{1 / 2}$ and $v_{y}=\left(\rho_{s y} / \chi\right)^{1 / 2}$, are then given by

$$
v_{x}=\left(\frac{-a^{2}\left\langle K_{x}\right\rangle_{\mathrm{MF}}}{4 \chi}\right)^{1 / 2}=v_{F},
$$




$$
v_{y}=\left(\frac{-b^{2}\left\langle K_{y}\right\rangle_{\mathrm{MF}}}{4 \chi}\right)^{1 / 2}=v_{\perp} .
$$

We find that the longitudinal velocity equals the Fermi velocity, because we have neglected the coupling of the spin-wave mode with the long-wavelength fluctuations. Taking into account the latter would lead to the renormalized spin-wave velocity $v_{x}=v_{F}(1-U N(0))^{1 / 2}$.

Finally, we note that we can carry out an appropriate rescaling of lengths to obtain an isotropic NL $\sigma \mathrm{M}$. Rescaling the lengths as $x^{\prime}=x / v_{x}, y^{\prime}=y / v_{y}$ and $\tau^{\prime}=\tau$, we obtain

$$
S_{\mathrm{spin}}^{\mathrm{eff}}=\frac{\sqrt{\rho_{s x} \rho_{s y}}}{2} \int_{\Lambda^{\prime}} d^{2} r^{\prime} d \tau^{\prime} \sum_{\mu}^{\tau^{\prime}, x^{\prime}, y^{\prime}}\left(\partial_{\mu} \mathbf{n}\right)^{2} .
$$

The cut-off $\Lambda^{\prime} \sim \Delta_{0}$ (in reciprocal space) is now isotropic. The dimensionless coupling constant of the isotropic $\mathrm{NL} \sigma \mathrm{M}$ (3.9) equals $\Delta_{0} /\left(\rho_{s x} \rho_{s y}\right)^{1 / 2}$. It is proportional to $\Delta_{0} \propto e^{-2 / U N(0)}$ and hence approaches zero in the weak-coupling limit $(U \rightarrow 0) 37$

\section{B. Berry phase term}

In general, in addition to the usual dynamical terms, the action describing antiferromagnetic spin systems in one or two dimensions is expected to have a topological Berry phase term. 36 Such a term was derived for antiferromagnetic systems described by the 2D isotropic Hubbard model by Wen et al 25 We now derive this term for our action.

The Berry phase term comes from the term proportional to $A_{0}^{z}$ in $\left\langle S^{\prime \prime}\right\rangle_{\mathrm{MF}}$. However, to get a non-zero result, it is not sufficient to retain only the long-wavelength part of the $R$ matrix, since the Berry phase term precisely results from the $2 k_{F}$ oscillating part. Instead of considering the more general structure of the $R$ matrix, we go back to the original tight-binding dispersion law, which is a more direct way for obtaining the Berry phase term. The term proportional to $A_{0}^{z}$ in $\left\langle S^{\prime \prime}\right\rangle_{\mathrm{MF}}$ then reads

$$
S_{\text {Berry }}=-\int d^{2} r d \tau A_{0}^{z}\left\langle\phi^{\dagger} \sigma_{z} \phi\right\rangle_{\mathrm{MF}},
$$

where $\left\langle\phi^{\dagger} \sigma_{z} \phi\right\rangle_{\mathrm{MF}}$ is the spin density in the mean-field state. Noting that $\left\langle\phi^{\dagger} \sigma_{z} \phi\right\rangle_{\mathrm{MF}}$ becomes $\left\langle\phi^{\dagger}\left(\tau_{0}+\tau_{+}+\right.\right.$ $\left.\left.\tau_{-}\right) \sigma_{z} \phi\right\rangle_{\mathrm{MF}}$ in the continuous formulation, we obtain, using the mean-field equations [Eq. (2.12)],

$$
\begin{aligned}
S_{\text {Berry }} & =-\int d^{2} r d \tau A_{0}^{z}\left\langle\phi^{\dagger}\left(\tau_{0}+\tau_{+}+\tau_{-}\right) \sigma_{z} \phi\right\rangle_{\mathrm{MF}} \\
& =-\frac{4 \Delta_{0}}{U} \int d^{2} r d \tau A_{0}^{z} \cos (\mathbf{Q} \cdot \mathbf{r}) .
\end{aligned}
$$

In general, it is not possible to express the field $A_{0}^{z}$ in terms of the $\mathbf{n}$ vector, because the former is a gaugedependent quantity. Nevertheless, it is still possible to express the variation of the $A_{0}^{z}$ field in terms of $\mathbf{n}$ as 25 $A_{0}^{z}=-i / 2 \int_{0}^{1} d \eta \mathbf{n}(\eta) \cdot\left(\partial_{\eta} \mathbf{n}(\eta) \times \partial_{\tau} \mathbf{n}(\eta)\right)$, where $\eta$ is an external parameter varying continuously from 0 to $1, \mathbf{n}(1)$ is the physical $\mathbf{n}$ field and $\mathbf{n}(0)=0$. The action can then be written as

$$
\begin{aligned}
S_{\text {Berry }}= & i \frac{2 \Delta_{0}}{U} \int d^{2} r d \tau \cos (\mathbf{Q} \cdot \mathbf{r}) \\
& \times \int_{0}^{1} d \eta \mathbf{n}(\eta) \cdot\left[\partial_{\eta} \mathbf{n}(\eta) \times \partial_{\tau} \mathbf{n}(\eta)\right] .
\end{aligned}
$$

Another equivalent way of writing the Berry term in the effective action is to express the gauge field $A_{0}^{z}$ in terms of the polar and azimuthal angles $\alpha$ and $\phi$ of the $\mathbf{n}$ vector. Using $A_{0}^{z}=-i / 2\left(1-\cos (\alpha(\tau, \mathbf{r})) \partial_{\tau} \phi(\tau, \mathbf{r})\right.$, the Berry term in the action then assumes the more familiar form 36

$$
\begin{aligned}
S_{\text {Berry }}= & i \frac{2 \Delta_{0}}{U} \int d^{2} r d \tau \cos (\mathbf{Q} \cdot \mathbf{r}) \\
& \times[1-\cos (\alpha(\tau, \mathbf{r}))] \partial_{\tau} \phi(\tau, \mathbf{r}) .
\end{aligned}
$$

For $2 \mathrm{D}$ and $3 \mathrm{D}$ antiferromagnets, it is generally believed that the presence of the Berry phase term plays no role. 36 The dynamics of the spin-wave mode is therefore determined by the $\mathrm{NL} \sigma \mathrm{M}$ [Eq. (3.4)] derived in the previous section.

\section{PHASON MODE}

The contribution to the phason effective action comes from the diamagnetic terms [Eqs. (2.35) and (2.38)], the chiral term $S_{J}$ [Eq. (2.47)], the interaction term $S_{I}$

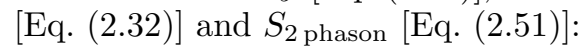

$$
\begin{aligned}
S_{\text {phason }}^{\mathrm{eff}}= & \frac{1}{\beta} \sum_{p_{n}} \int \frac{d^{2} p}{(2 \pi)^{2}}\left\{\sum_{\mu \nu}^{0, x, y} \frac{1}{2}\left[\left(e A_{\mu}\left(i p_{n}, \mathbf{p}\right)+i \delta \rho^{\mathrm{HS}}\left(i p_{n}, \mathbf{p}\right) \delta_{\mu 0}\right) P_{\mu \nu}\left(i p_{n}, \mathbf{p}\right)\left(e A_{\nu}\left(-i p_{n},-\mathbf{p}\right)+i \delta \rho^{\mathrm{HS}}\left(-i p_{n},-\mathbf{p}\right) \delta_{\nu 0}\right)\right]\right. \\
& +\sum_{\mu}^{0, x, y}\left[\left(e A_{\mu}\left(i p_{n}, \mathbf{p}\right)+i \delta \rho^{\mathrm{HS}}\left(i p_{n}, \mathbf{p}\right) \delta_{\mu 0}\right) F_{\mu}\left(i p_{n}, \mathbf{p}\right) \theta\left(-i p_{n},-\mathbf{p}\right)\right]+\frac{1}{2}\left|\theta\left(i p_{n}, \mathbf{p}\right)\right|^{2} D\left(i p_{n}, \mathbf{p}\right) \\
& \left.+i \delta \rho\left(i p_{n}, \mathbf{p}\right) \delta \rho^{\mathrm{HS}}\left(-i p_{n},-\mathbf{p}\right)+\frac{U}{4}\left|\delta \rho\left(i p_{n}, \mathbf{p}\right)\right|^{2}\right\}
\end{aligned}
$$

where the polarization tensor $P_{\mu \nu}$ and the coupling coefficients $F_{\mu}$ and $D$, are given by 


$$
\begin{aligned}
P_{\mu \nu}\left(i p_{n}, \mathbf{p}\right) & =\left(\begin{array}{ccc}
-\frac{N(0)}{3 \Delta_{0}^{2}}\left(v_{F}^{2} p_{x}^{2}+v_{\perp}^{2} p_{y}^{2}\right) & -\frac{N(0) v_{F}^{2}}{3 \Delta_{0}^{2}} i p_{n} p_{x} & -\frac{N(0) v_{\perp}^{2}}{3 \Delta_{0}^{2}} i p_{n} p_{y} \\
-\frac{N(0) v_{F}^{2}}{3 \Delta_{0}^{2}} i p_{n} p_{x} & \frac{N(0) v_{F}^{2}}{3 \Delta_{0}^{2}} p_{n}^{2} & 0 \\
-\frac{N(0) v_{\perp}^{2}}{3 \Delta_{0}^{2}} i p_{n} p_{y} & 0 & \frac{N(0) v_{\perp}^{2}}{3 \Delta_{0}^{2}} p_{n}^{2}
\end{array}\right), \\
F_{\mu}\left(i p_{n}, \mathbf{p}\right) & =\left(i N(0) v_{F} p_{x},-N(0) v_{F} p_{n}, 0\right) \\
D\left(i p_{n}, \mathbf{p}\right) & =\frac{N(0)}{2}\left(p_{n}^{2}+v_{F}^{2} p_{x}^{2}+v_{\perp}^{2} p_{y}^{2}\right) .
\end{aligned}
$$

The contribution from the diamagnetic terms in Eqs. (2.35) and (2.38) exactly cancels the gauge-

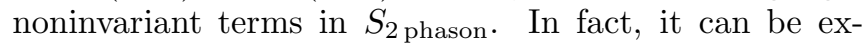
plicitly checked that the polarization tensor $P_{\mu \nu}$ is transverse. Also, we note that the phase field $\theta$ couples to the (gauge-invariant) electric field $E_{x}=-p_{n} A_{x}+i p_{x} A_{0}$. This, together with the transverse polarization tensor $P_{\mu \nu}$, ensures that if we integrate out the fields $\delta \rho, \delta \rho^{\mathrm{HS}}$ and $\theta$ to obtain an effective action $S^{\text {eff }}\left[A_{\mu}\right]$, it will be gauge-invariant. Since the coupling term between $\theta$ and $A_{0}$ comes from $S_{J}$, it turns out that the contribution from the Jacobian of the chiral anomaly is crucial for obtaining a gauge-invariant effective action.

\section{A. Equations of motion}

We consider the electromagnetic field as being the external field, i.e. we neglect the phason-polariton mode. 38 Varying the effective action with respect to the fields $\theta$, $\rho$ and $\rho^{\mathrm{HS}}$, we obtain the three coupled equations

$$
\begin{array}{r}
D\left(i p_{n}, \mathbf{p}\right) \theta\left(i p_{n}, \mathbf{p}\right)+\sum_{\mu}^{0, x, y} F_{\mu}\left(i p_{n}, \mathbf{p}\right)\left(e A_{\mu}\left(i p_{n}, \mathbf{p}\right)\right. \\
\left.+i \delta \rho^{\mathrm{HS}}\left(i p_{n}, \mathbf{p}\right) \delta_{\mu 0}\right)=0 \\
F_{0}\left(-i p_{n},-\mathbf{p}\right) \theta\left(i p_{n}, \mathbf{p}\right)+\delta \rho\left(i p_{n}, \mathbf{p}\right) \\
+\sum_{\mu}^{0, x, y} P_{0 \mu}\left(i p_{n}, \mathbf{p}\right)\left(e A_{\mu}\left(i p_{n}, \mathbf{p}\right)+i \delta \rho^{\mathrm{HS}}\left(i p_{n}, \mathbf{p}\right) \delta_{\mu 0}\right)=0 \\
\frac{U}{2} \delta \rho\left(i p_{n}, \mathbf{p}\right)+i \delta \rho^{\mathrm{HS}}\left(i p_{n}, \mathbf{p}\right)=0 .
\end{array}
$$

Using Eq. (4.5), we can eliminate the HS field $\delta \rho^{\mathrm{HS}}$. This gives two coupled equations for the physical fields $\delta \rho$ and $\theta$. Substituting the expressions for $D, P_{\mu \nu}$ and $F_{\mu}$ from Eq. (4.2), and retaining terms up to second order in frequency and momenta, we get

$$
\begin{gathered}
\left(p_{n}^{2}+v_{F}^{2} p_{x}^{2}+v_{\perp}^{2} p_{y}^{2}\right) \theta\left(i p_{n}, \mathbf{p}\right) \\
-i v_{F} p_{x} U \delta \rho\left(i p_{n}, \mathbf{p}\right)+2 e v_{F} E_{x}\left(i p_{n}, \mathbf{p}\right)=0,
\end{gathered}
$$

$$
v_{F} N(0) p_{x} \theta\left(i p_{n}, \mathbf{p}\right)+i \delta \rho\left(i p_{n}, \mathbf{p}\right)=0 .
$$

Thus, we obtain the well-known relation between charge and phase fluctuations:

$$
\delta \rho(\tau, \mathbf{r})=v_{F} N(0) \partial_{x} \theta(\tau, \mathbf{r})=\frac{1}{\pi b} \partial_{x} \theta(\tau, \mathbf{r}) .
$$

From (4.6), we finally obtain the (decoupled) equations of motion

$$
\begin{aligned}
\left(\partial_{t}^{2}-v_{\mathrm{ph}}^{2} \partial_{x}^{2}-v_{\perp}^{2} \partial_{y}^{2}\right) \theta(t, \mathbf{r}) & =-2 e v_{F} E_{x}(t, \mathbf{r}), \\
\left(\partial_{t}^{2}-v_{\mathrm{ph}}^{2} \partial_{x}^{2}-v_{\perp}^{2} \partial_{y}^{2}\right) \delta \rho(t, \mathbf{r}) & =-\frac{2 e v_{F}}{\pi b} \partial_{x} E_{x}(t, \mathbf{r}),
\end{aligned}
$$

where we have Wick-rotated back to real time $t$, and $v_{\mathrm{ph}}=v_{F}(1+U N(0))^{1 / 2}$ is the renormalized phason velocity. This renormalization of the longitudinal phason velocity has been obtained previously from a more conventional approach based on Green function calculations.1

\section{B. Effective action $S\left[\theta, A_{\mu}\right]$}

In this section, we derive the effective action $S^{\operatorname{eff}}\left[\theta, A_{\mu}\right]$ for the phase mode.

If we try to integrate out the HS fields $\delta \rho$ and $\delta \rho^{\mathrm{HS}}$ in $S_{\text {phason }}^{\text {eff }}\left[\delta \rho, \delta \rho^{\mathrm{HS}}, \theta, A_{\mu}\right]$, we immediately face the problem of inverting the coefficient $P_{00}$ of the quadratic term in $\delta \rho^{\mathrm{HS}}$, since $P_{00}^{-1}$ is singular at $p_{n}, \mathbf{p}=0$. Nevertheless, we note from the results of the last section, that this term does not contribute to the equations of motion up to quadratic order in external momenta and frequency. We can therefore ignore this term while integrating out the field $\delta \rho^{\mathrm{HS}}$. As we shall see, the effective action so obtained, although not exact, reproduces the correct equations of motion for the phase field $\theta$.

Integration of the $\delta \rho^{\mathrm{HS}}$ fields within the above-stated approximation then gives a factor of $\prod_{p_{n}, \mathbf{p}} \delta(\delta \rho-$ $\left.i p_{x} \theta / \pi b\right)$ and we are left with the partition function

$$
Z=\int D \delta \rho D \theta D A_{\mu} \delta\left(\delta \rho-\frac{i}{\pi b} p_{x} \theta\right) e^{-S^{\mathrm{eff}}\left[\delta \rho, A_{\mu}, \theta\right]},
$$

with the action 


$$
\begin{aligned}
S^{\mathrm{eff}}\left[\delta \rho, A_{\mu}, \theta\right]= & \frac{1}{\beta} \sum_{p_{n}} \int \frac{d^{2} p}{(2 \pi)^{2}}\left\{\sum_{\mu \nu}^{0, x, y} \frac{1}{2}\left[e A_{\mu}\left(i p_{n}, \mathbf{p}\right) P_{\mu \nu}\left(i p_{n}, \mathbf{p}\right) e A_{\nu}\left(-i p_{n},-\mathbf{p}\right)\right]\right. \\
& \left.+\sum_{\mu}^{0, x, y}\left[e A_{\mu}\left(i p_{n}, \mathbf{p}\right) F_{\mu}\left(i p_{n}, \mathbf{p}\right) \theta\left(-i p_{n},-\mathbf{p}\right)\right]+\frac{1}{2}\left|\theta\left(i p_{n}, \mathbf{p}\right)\right|^{2} D\left(i p_{n}, \mathbf{p}\right)+\frac{U}{4}\left|\delta \rho\left(i p_{n}, \mathbf{p}\right)\right|^{2}\right\} .
\end{aligned}
$$

Integrating out $\delta \rho$, one then gets the effective action

$$
\begin{aligned}
S^{\mathrm{eff}}\left[\theta, A_{\mu}\right]= & \frac{1}{\beta} \sum_{p_{n}} \int \frac{d^{2} p}{(2 \pi)^{2}}\left\{\sum_{\mu \nu}^{0, x, y} \frac{1}{2}\left[e A_{\mu}\left(i p_{n}, \mathbf{p}\right) P_{\mu \nu}\left(i p_{n}, \mathbf{p}\right) e A_{\nu}\left(-i p_{n},-\mathbf{p}\right)\right]\right. \\
& \left.+e N(0) v_{F} E_{x}\left(i p_{n}, \mathbf{p}\right) \theta\left(-i p_{n},-\mathbf{p}\right)+\frac{1}{2}\left|\theta\left(i p_{n}, \mathbf{p}\right)\right|^{2} D^{\prime}\left(i p_{n}, \mathbf{p}\right)\right\}
\end{aligned}
$$

where $D^{\prime}\left(i p_{n}, \mathbf{p}\right)=N(0)\left(p_{n}^{2}+v_{\mathrm{ph}}^{2} p_{x}^{2}+v_{\perp}^{2} p_{y}^{2}\right) / 2$ is the effective inverse phason propagator. This clearly demonstrates the modification of the phason velocity due to the interaction between the phase field $\theta$ and the density fluctuation $\delta \rho$. It is easy to check, by varying Eq. (4.11) with respect to $\theta$, that this effective action reproduces the same equation of motion for $\theta$ as Eq. (4.6) derived in the earlier section.

\section{Effective action $S\left[\delta \rho, A_{\mu}\right]$}

To obtain the effective action $S\left[\delta \rho, A_{\mu}\right]$ for the density fluctuations $\delta \rho$, we start from the effective action $S^{\text {eff }}\left[\delta \rho, \theta, A_{\mu}\right]$ [Eq. (4.10)], and integrate out the phase field $\theta$. One then obtains the effective action

$$
\begin{aligned}
S\left[\delta \rho, A_{\mu}\right]= & \frac{1}{\beta} \sum_{p_{n}} \int \frac{d^{2} p}{(2 \pi)^{2}}\left\{\sum_{\mu \nu}^{0, x, y} \frac{1}{2}\left[e A_{\mu}\left(i p_{n}, \mathbf{p}\right) P_{\mu \nu}\left(i p_{n}, \mathbf{p}\right) e A_{\nu}\left(-i p_{n},-\mathbf{p}\right)\right]\right. \\
& \left.-e\left(A_{0}\left(i p_{n}, \mathbf{p}\right)-\frac{p_{n}}{i p_{x}} A_{x}\left(i p_{n}, \mathbf{p}\right)\right) \delta \rho\left(-i p_{n},-\mathbf{p}\right)+\frac{1}{2}\left|\delta \rho\left(i p_{n}, \mathbf{p}\right)\right|^{2} \chi_{\rho \rho}^{-1}\right\},
\end{aligned}
$$

where we have introduced the density-density correlation function

$$
\begin{aligned}
\chi_{\rho \rho}\left(i p_{n}, \mathbf{p}\right) & =\left\langle\delta \rho\left(i p_{n}, \mathbf{p}\right) \delta \rho\left(-i p_{n},-\mathbf{p}\right)\right\rangle \\
& =2 N(0) \frac{v_{F}^{2} p_{x}^{2}}{p_{n}^{2}+v_{\mathrm{ph}}^{2} p_{x}^{2}+v_{\perp}^{2} p_{y}^{2}} .
\end{aligned}
$$

It is easy to see from the expression of the density-density correlation function that the phason does not induce any transverse density fluctuations.

The currents in the longitudinal and transverse directions are given by

$$
\begin{aligned}
& j_{x}\left(i p_{n}, \mathbf{p}\right)=-\left.\frac{\delta S}{\delta A_{x}\left(-i p_{n},-\mathbf{p}\right)}\right|_{A_{\mu}=0}=-\frac{e p_{n}}{i p_{x}} \delta \rho\left(i p_{n}, \mathbf{p}\right), \\
& j_{y}\left(i p_{n}, \mathbf{p}\right)=-\left.\frac{\delta S}{\delta A_{y}\left(-i p_{n},-\mathbf{p}\right)}\right|_{A_{\mu}=0}=0
\end{aligned}
$$

These expressions agree with the (real time) continuity equation $\partial_{t} \rho+\nabla \cdot \mathbf{j}=0$. Also, we see that there is no current due to the phason mode in the $y$ direction. The contribution to the current across the chain comes entirely from the quasi-particle excitations 39 From Eqs. (4.7) and (4.14), we obtain (in real time)

$$
j_{x}(t, \mathbf{r})=-\frac{e}{\pi b} \partial_{t} \theta(t, \mathbf{r})
$$

Note that the expressions of the currents as a function of the phase $\theta$ [Eqs. (4.7) and (4.15)] have been obtained to lowest order in a gradient expansion. They have been recently generalized to higher order in Ref. 40.

The current-current correlators can also be obtained from the effective action. Using Eqs. (4.13) and (4.14), we find that the current-current correlator $\chi_{j_{\mu} j_{\nu}}=\left\langle j_{\mu} j_{\nu}\right\rangle$ can be expressed in terms of the density-density correlation function as

$$
\begin{aligned}
\chi_{j_{x} j_{x}}\left(i p_{n}, \mathbf{p}\right) & =-\frac{e^{2} p_{n}^{2}}{p_{x}^{2}} \chi_{\rho \rho}\left(i p_{n}, \mathbf{p}\right) \\
& =-2 N(0) e^{2} \frac{v_{F}^{2} p_{n}^{2}}{p_{n}^{2}+v_{\mathrm{ph}}^{2} p_{x}^{2}+v_{\perp}^{2} p_{y}^{2}}, \\
\chi_{j_{y} j_{y}}\left(i p_{n}, \mathbf{p}\right) & =\chi_{j_{x} j_{y}}\left(i p_{n}, \mathbf{p}\right)=0 .
\end{aligned}
$$

From the expression of the current-current correlator, we then obtain the ac conductivity $\sigma_{x x}(\omega)$ as

$$
\begin{aligned}
\sigma_{x x}(\omega) & =-\left.\frac{1}{p_{n}} \chi_{j_{x} j_{x}}\left(i p_{n}, \mathbf{p}=0\right)\right|_{i p_{n}=\omega+i 0^{+}} \\
& =2 N(0) e^{2} v_{F}^{2}\left(i \mathcal{P}\left(\frac{1}{\omega}\right)+\pi \delta(\omega)\right)
\end{aligned}
$$


where $\mathcal{P}$ denotes the principal part. The real part of the conductivity therefore satisfies the f-sum rule

$$
\int_{-\infty}^{\infty} \operatorname{Re}\left[\sigma_{x x}(\omega)\right] d \omega=\frac{\omega_{p}^{2}}{4}
$$

where $\omega_{p}=\left(8 e^{2} v_{F} / b\right)^{1 / 2}$ is the plasma frequency. The phason mode completely exhausts the spectral weight and we obtain the well-known result that there is no contribution to the longitudinal conductivity from the quasi-particle excitations in a clean SDW system.

\section{CONCLUSION}

We have derived the effective action for the low-energy collective modes of quasi-1D SDW systems. The introduction of a fluctuating spin-quantization axis in the functional integral allows to consider the phason mode, the spin-wave mode, and the long-wavelength charge fluctuations on equal footing.

We find that the spin-wave mode is governed by an isotropic NL $\sigma \mathrm{M}$ together with a topological Berry phase term. By a suitable length rescaling, one can obtain an isotropic NL $\sigma \mathrm{M}$ with an effective dimensionless coupling constant $\Delta_{0} /\left(\rho_{s x} \rho_{s y}\right)^{1 / 2}$, where $\rho_{s x}$ and $\rho_{s y}$ are the spinstiffness coefficients. The coupling constant, proportional to the mean-field gap $\Delta_{0} \propto e^{-2 / U N(0)}$, is small in the weak coupling limit $U N(0) \ll 1$.

The sliding mode is governed by an effective Lagrangian $\mathcal{L}(\theta, \rho)$ which is a function of two independent fields: the phase $\theta$ of the SDW condensate and the charge density field $\rho$. From the coupled equations satisfied by these two variables, we obtain the phason dynamics and its contribution to the current-current and densitydensity correlation functions.

\section{ACKNOWLEDGMENT}

One of the authors (KS) would like to thank Victor Yakovenko for helpful discussions and for support during the work. Laboratoire de Physique des Solides is associé au CNRS.

\section{APPENDIX A:}

In this section, we sketch the calculation of the current-current correlators. These correlators are given by

$$
\Pi_{j_{\mu}^{\nu} j_{\mu^{\prime}}^{\prime}}\left(i p_{n}, \mathbf{p}\right) \equiv \Pi_{j j^{\prime}}\left(i p_{n}, \mathbf{p}\right)=\left\langle j\left(i p_{n}, \mathbf{p}\right) j^{\prime}\left(-i p_{n},-\mathbf{p}\right)\right\rangle_{\mathrm{MF}}
$$

The currents $j_{\mu}^{\nu}$ (or $j$ in the simplified notation) can be defined using Eq. (2.25) as

$$
j\left(i p_{n}, \mathbf{p}\right)=\frac{1}{\sqrt{\beta}} \sum_{\omega_{n}} \int \frac{d^{2} k}{(2 \pi)^{2}} \sum_{\alpha}^{+-} \sum_{\sigma \sigma^{\prime}}^{\uparrow \downarrow} \phi_{\alpha \sigma^{\prime}}^{\dagger}\left(i \omega_{n}, \mathbf{k}\right) v_{\alpha \sigma \sigma^{\prime}}\left(k_{y}, k_{y}+p_{y}\right) \phi_{\alpha \sigma}\left(i \omega_{n}+i p_{n}, \mathbf{k}+\mathbf{p}\right)
$$

where we have set the area of the system to unity. The functions $v_{\alpha \sigma \sigma^{\prime}}\left(k_{y}, k_{y}+p_{y}\right)$ are the current operators which can be easily read off from the definition of the currents in Eq. 2.25). For example, for the charge current in the $x$ direction $j_{x}^{0}, v_{\alpha \sigma \sigma^{\prime}}\left(k_{y}, k_{y}+p_{y}\right)=v_{F} \alpha \delta_{\sigma \sigma^{\prime}}$. From A2, we then deduce

$$
\begin{aligned}
\Pi_{j j^{\prime}}\left(i p_{n}, \mathbf{p}\right)= & -\frac{1}{\beta} \sum_{\omega_{n}} \int \frac{d^{2} k}{(2 \pi)^{2}} \sum_{\alpha}^{+-} \sum_{\sigma \sigma^{\prime}}^{\uparrow \downarrow}\left[v_{\alpha \sigma \sigma^{\prime}}\left(k_{y}-p_{y}, k_{y}\right) v_{\alpha \sigma^{\prime} \sigma}^{\prime}\left(k_{y}, k_{y}-p_{y}\right) G_{\alpha \sigma^{\prime}}\left(i \omega_{n}, \mathbf{k}\right) G_{\alpha \sigma}\left(i \omega_{n}-i p_{n}, \mathbf{k}-\mathbf{p}\right)\right. \\
& \left.+v_{\alpha \sigma \sigma^{\prime}}\left(k_{y}-p_{y}, k_{y}\right) v_{\bar{\alpha} \sigma^{\prime} \sigma}^{\prime}\left(k_{y}+\pi / b, k_{y}-p_{y}+\pi / b\right) F_{\alpha \sigma^{\prime}}\left(i \omega_{n}, \mathbf{k}\right) F_{\bar{\alpha} \sigma}\left(i \omega_{n}-i p_{n}, \mathbf{k}-\mathbf{p}-\alpha \mathbf{Q}\right)\right]
\end{aligned}
$$

where $\bar{\alpha}=\mp$ for $\alpha= \pm$ and $G_{ \pm \sigma}$ and $F_{ \pm \sigma}$ are the mean-field propagators. We evaluate the frequency sum and the $k_{x}$ integral using $\epsilon_{\alpha}(\mathbf{k}-\alpha \mathbf{Q})=-\epsilon_{\bar{\alpha}}(\mathbf{k})$ and expanding the energy dispersion $\epsilon_{\alpha}$ in powers of external momenta:

$$
\begin{aligned}
\epsilon_{\alpha}(\mathbf{k})-\epsilon_{\alpha}(\mathbf{k}-\mathbf{p}) & =\epsilon_{x}+\epsilon_{y} \\
\epsilon_{y} & =2 t_{b} b p_{y} \sin \left(k_{y} b\right)-t_{b} b^{2} p_{y}^{2} \cos \left(k_{y} b\right)+O\left(p_{y}^{3}\right), \\
\epsilon_{x} & =\alpha v_{F} p_{x} .
\end{aligned}
$$

Using these relations and Eq. 2.16) one can evaluate the frequency sum and the $k_{x}$ integral up to second order in $p_{n}$ and $\epsilon_{\mathrm{T}}=\epsilon_{x}+\epsilon_{y}\left(O\left(p_{n}^{2}, \epsilon_{\mathrm{T}}^{2}, p_{n} \epsilon_{\mathrm{T}}\right)\right)$ to obtain 


$$
\begin{gathered}
\frac{1}{\beta} \sum_{\omega_{n}} \int \frac{d k_{x}}{2 \pi} F_{\alpha \sigma^{\prime}}\left(i \omega_{n}, \mathbf{k}\right) F_{\bar{\alpha} \sigma}\left(i \omega_{n}-i p_{n}, \mathbf{k}-\mathbf{p}-\alpha \mathbf{Q}\right)=\operatorname{sgn}(\sigma) \operatorname{sgn}\left(\sigma^{\prime}\right) \frac{N(0)}{2}\left(\frac{1}{2}-\frac{p_{n}^{2}+\epsilon_{\mathrm{T}}^{2}}{12 \Delta_{0}^{2}}\right), \\
\frac{1}{\beta} \sum_{\omega_{n}} \int \frac{d k_{x}}{2 \pi} G_{\alpha \sigma^{\prime}}\left(i \omega_{n}, \mathbf{k}\right) G_{\alpha \sigma}\left(i \omega_{n}-i p_{n}, \mathbf{k}-\mathbf{p}\right)=-\frac{N(0)}{2}\left(\frac{1}{2}-\frac{p_{n}^{2}-\epsilon_{\mathrm{T}}^{2}}{12 \Delta_{0}^{2}}+\frac{i p_{n} \epsilon_{\mathrm{T}}}{6 \Delta_{0}^{2}}\right) .
\end{gathered}
$$

Substituting Eq. (A5) in the expression for the correlator [Eq. (A3]] we get

$$
\begin{aligned}
\Pi_{j j^{\prime}}\left(i p_{n}, \mathbf{p}\right)= & \frac{N(0)}{2} \int \frac{d k_{y}}{2 \pi} \sum_{\alpha}^{+-} \sum_{\sigma \sigma^{\prime}}^{\uparrow \downarrow}\left[v_{\alpha \sigma \sigma^{\prime}}\left(k_{y}-p_{y}, k_{y}\right) v_{\alpha \sigma^{\prime} \sigma}^{\prime}\left(k_{y}, k_{y}-p_{y}\right)\left(\frac{1}{2}-\frac{p_{n}^{2}-\epsilon_{\mathrm{T}}^{2}}{12 \Delta_{0}^{2}}+\frac{i p_{n} \epsilon_{\mathrm{T}}}{6 \Delta_{0}^{2}}\right)\right. \\
& \left.+v_{\alpha \sigma \sigma^{\prime}}\left(k_{y}-p_{y}, k_{y}\right) v_{\bar{\alpha} \sigma^{\prime} \sigma}^{\prime}\left(k_{y}+\pi / b, k_{y}-p_{y}+\pi / b\right) \operatorname{sgn}(\sigma) \operatorname{sgn}\left(\sigma^{\prime}\right)\left(-\frac{1}{2}+\frac{p_{n}^{2}+\epsilon_{\mathrm{T}}^{2}}{12 \Delta_{0}^{2}}\right)\right] .
\end{aligned}
$$

The dependence on $k_{y}$ comes from $\epsilon_{y}$ and $v_{\alpha \sigma \sigma^{\prime}}$. The latter is either $k_{y}$ independent or depends on $k_{y}$ through the function $f=\sin \left(\left(k_{y}-p_{y}\right) b\right)+\sin \left(k_{y} b\right)=2 \sin \left(k_{y} b\right)-p_{y} b \cos \left(k_{y} b\right)-p_{y}^{2} b^{2} \sin \left(k_{y} b\right) / 2+O\left(p_{y}^{3}\right)$. To evaluate the $k_{y}$ integral in (A6), we therefore need only a limited set of integrals, which, up to order $p_{y}^{2}$, are given by

$$
\begin{aligned}
\langle f\rangle_{k_{y}} & =\left\langle\epsilon_{y}\right\rangle_{k_{y}}=\left\langle f \epsilon_{y}^{2}\right\rangle_{k_{y}}=\left\langle f^{2} \epsilon_{y}\right\rangle_{k_{y}}=0, \\
\left\langle f^{2}\right\rangle_{k_{y}} & =2-\frac{p_{y}^{2} b^{2}}{2}, \quad\left\langle\epsilon_{y}^{2}\right\rangle_{k_{y}}=v_{\perp}^{2} p_{y}^{2}, \quad\left\langle f \epsilon_{y}\right\rangle_{k_{y}}=2 t_{b} b p_{y},\left\langle f^{2} \epsilon_{y}^{2}\right\rangle_{k_{y}}=3 v_{\perp}^{2} p_{y}^{2},
\end{aligned}
$$

where we have used the short-hand notation $\langle\cdots\rangle_{k_{y}}$ for $\int d k_{y} /(2 \pi) \cdots$.

From Eqs. A6) and (A7), it is then a matter of straightforward algebra to obtain the different correlators. As an illustrative example we sketch the calculation of $\Pi_{j_{y}^{0} j_{y}^{0}}$. Since the current operator in this case is given by $v_{\alpha \sigma \sigma^{\prime}}\left(k_{y}, k_{y}-\right.$ $\left.p_{y}\right)=t_{b} b \delta_{\sigma \sigma^{\prime}} f\left(k_{y}, p_{y}\right)$, we obtain

$$
\begin{aligned}
\Pi_{j_{y}^{0} j_{y}^{0}}\left(i p_{n}, \mathbf{p}\right) & =\frac{N(0) t_{b}^{2} b^{2}}{2} \sum_{\alpha}^{+-} \sum_{\sigma \sigma^{\prime}}^{\uparrow \downarrow} \delta_{\sigma \sigma^{\prime}}\left\langle f^{2}\left(1-\frac{p_{n}^{2}}{6 \Delta_{0}^{2}}+\frac{i p_{n} \epsilon_{\mathrm{T}}}{6 \Delta_{0}^{2}}\right)\right\rangle_{k_{y}} \\
& =2 N(0) v_{\perp}^{2}\left(1-\frac{p_{y}^{2} b^{2}}{4}-\frac{p_{n}^{2}}{6 \Delta_{0}^{2}}\right),
\end{aligned}
$$

where we have used Eq. (A7) to evaluate the $k_{y}$ integrals to obtain the last line and retained only terms up to second order in external frequency and momenta.

The other correlators can be obtained following an exactly similar procedure and in this way, we finally obtain Eqs. (2.50) for the charge and spin current-current correlators.

${ }^{1}$ For reviews on DW systems, see G. Grüner, Density Waves in Solids (Addison-Wesley, New York, 1994); Rev. Mod. Phys. 60, 1129 (1988); Rev. Mod. Phys. 66, 1 (1994).

${ }^{2}$ H. Fröhlich, Proc. Roy. Soc. Lond. A 223, 296 (1954).

${ }^{3}$ R.E. Peierls, Quantum Theory of Solids (Oxford University Press, New York, 1955).

${ }^{4}$ A.W. Overhauser, Phys. Rev. 128, 1437 (1962).

${ }^{5}$ P.A. Lee, T.M. Rice, and P.W. Anderson, Solid State Comm. 14, 703 (1974).

${ }^{6}$ H. Fukuyama, J. Phys. Soc. Japan 41, 513 (1976).

${ }^{7}$ S. Brazovski and I. Dzyaloshinskii, JETP 44, 1233 (1976). Note that these authors perform a chiral transformation without computing the Jacobian (chiral anomaly) so that some parameters in their Lagrangian have incorrect values.
[The chiral anomaly (i.e. the existence of a non trivial Jacobian of the chiral transformation) was not known at that time.]

${ }^{8}$ H. Fukuyama and P.A. Lee, Phys. Rev. B 17, 535 (1978).

${ }^{9}$ P.A. Lee and T.M. Rice, Phys. Rev. B 19, 3970 (1979).

${ }^{10}$ G.C. Psaltakis, Solid State Comm. 51, 535 (1984).

${ }^{11}$ K. Maki and A. Virosztek, Phys. Rev. B 41, 557 (1990).

${ }^{12}$ K. Maki and A. Virosztek, Phys. Rev. B 42, 655 (1990).

13 S. Brazovskii, J. Physique I 3, 2417 (1993).

${ }^{14}$ D. Poilblanc and P. Lederer, Phys. Rev. B 37, 9650 (1987); Phys. Rev. B 37, 9672 (1987).

15 A. Bjelis and D. Zanchi, Phys. Rev. B 49, 5968 (1994).

${ }^{16}$ K. Maki and A. Virosztek, Phys. Rev. B 36, 511 (1987).

${ }^{17}$ K. Takano, Prog. Theor. Phys. 68, 1 (1982),

${ }^{18}$ I.V. Krive and A.S. Rozhavsky, Phys. Lett. A113, 313 (1985).

19 Z. Su and B. Sakita, Phys. Rev. Lett. 56, 780 (1986); Phys. Rev. B 38, 7421 (1988); B. Sakita and Z. Su, Prog. Theor. Phys. 86, 238 (1986). 
${ }^{20}$ M. Ishikawa and H. Takayama, Prog. Theor. Phys. 79, 359 (1988).

${ }^{21}$ Y. Suzumura, J. Phys. Soc. Japan 59, 1711 (1990).

${ }^{22}$ M. Girard, Mémoire de maîtrise, Université de Sherbrooke, 1993 (unpublished).

${ }^{23}$ N. Nagaosa and M. Oshikawa, J. Phys. Soc. Japan 65, 2241 (1996); A. Tanaka and M. Machida, J. Phys. Soc. Japan 67, 748 (1998).

${ }^{24}$ V.M. Yakovenko and H.S. Goan, Phys. Rev. B 58, 10648 (1998).

${ }^{25}$ S. Wen and A. Zee, Phys. Rev. Lett. 61, 1025 (1988).

${ }^{26}$ H.J. Schulz, Phys. Rev. Lett. 65, 2462 (1990); H.J. Schulz in The Hubbard Model, edited by D. Baeriswyl et al., (Plenum Press, New York, 1995).

${ }^{27}$ Z.Y. Weng, C.S. Ting and T.K. Lee, Phys. Rev. B 43, 3790 (1991).

${ }^{28}$ To be more specific, the interacting part of the Hamil tonian can be written in the g-ology formulation as 22 $H_{I}=\frac{1}{2} \sum_{n} \int d x\left[\left(2 g_{1}-g_{2}\right) O_{n}^{\dagger}(x) O_{n}(x)-g_{2} \mathbf{O}_{n}^{\dagger}(x) \mathbf{O}_{n}(x)\right]$, where $O=\sum_{\sigma} \psi_{-\sigma}^{\dagger} \psi_{+\sigma}$ and $\mathbf{O}=\sum_{\sigma, \sigma^{\prime}} \psi_{-\sigma}^{\dagger} \boldsymbol{\sigma}_{\sigma, \sigma^{\prime}} \psi_{+\sigma^{\prime}}$ are the charge and spin densities, respectively (we use the notations introduced in Sec. III). $g_{1}=g_{2}=U$ in the Hubbard model. This form of the Hamiltonian obviously preserves spin rotation invariance, and also allows to recover the correct mean-field solution from a saddle peint approximation within a functional integral formalism.22 However, it does not take into account the long-wavelength charge or spin fluctuations.

${ }^{29}$ It has been argued that in a SDW system the thermally ex- cited electrons do not screen the Coulomb interaction. As a result, the longitudinal phason is completely absorbed by the plasmon due to the Anderson-Higgs mechanism: A. Virosztek and K. Maki, Phys. Rev. B 49, 6074 (1994).

${ }^{30}$ S. De Palo, C. Castellani, C. Di Castro and B.K. Chakraverty, Phys. Rev. B 60, 564 (1999).

${ }^{31}$ Note that this limit is required for the behavior of the system to be trully 2D. The condition $\Delta_{0} \gtrsim t_{b}$ would imply a 1D behavior (with a weak Josephson-like interchain coupling), a regime where the mean-field approach used in this paper breaks down.

${ }^{32}$ K. Fujikawa, Phys. Rev. Lett. 42, 1195 (1979).

${ }^{33}$ Note that by using the mean-field propagators, we assume that $\delta \rho$ depends only on $\delta \theta$ (and not on $\theta$ ).

${ }^{34}$ We could also expect a contribution $-\int_{0}^{\beta} d^{2} r d \tau \rho \delta A_{0}^{0}$, where $\rho=\partial_{x} \theta$ [see Eq. (2.45)]. According to Ref. 24, this term does not contribute to the chiral anomaly.

35 A.M.J. Schakel, cond-mat/9805152, May 13 (1998).

${ }^{36} \mathrm{~A}$. Auerbach, Interacting Electrons and Quantum Magnetism (Springer Verlag, New York, 1994).

37 This point has also been noted by Schulz in the context of the isotropic 2D Hubbard model.26

38 P.B. Littlewood, Phys. Rev. B 36, 3108 (1987); S.N. Artemenko and W. Wonneberger, J. Phys. I France 6, 2079 (1996).

39 A. Virosztek, B. Dora, and K. Maki, Europhys. Lett. 47, 358 (1999).

40 A.S. Rozhavsky, Y.V. Pershin, and I.A. Romanovsky, Europhys. Lett. 46, 50 (1999). 